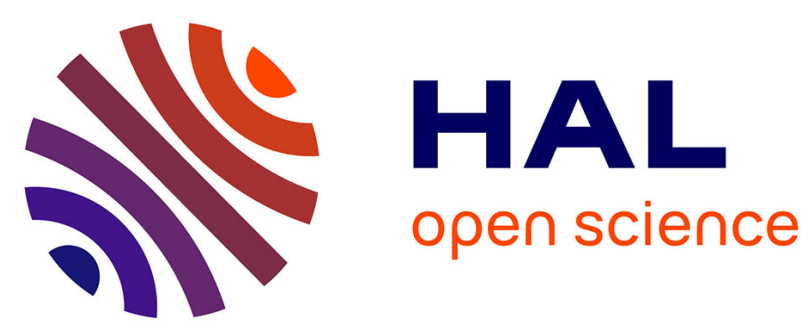

\title{
A trade-off between productivity and cost for the integrated part quality inspection and preventive maintenance planning under uncertainty
}

Mohammad Rezaei-Malek, Ali Siadat, Jean-Yves Dantan, Reza

Tavakkoli-Moghaddam

\section{To cite this version:}

Mohammad Rezaei-Malek, Ali Siadat, Jean-Yves Dantan, Reza Tavakkoli-Moghaddam. A trade-off between productivity and cost for the integrated part quality inspection and preventive maintenance planning under uncertainty. International Journal of Production Research, 2018, 57 (19), pp.59515973. 10.1080/00207543.2018.1556411 . hal-02436389

\section{HAL Id: hal-02436389 \\ https://hal.science/hal-02436389}

Submitted on 13 Jan 2020

HAL is a multi-disciplinary open access archive for the deposit and dissemination of scientific research documents, whether they are published or not. The documents may come from teaching and research institutions in France or abroad, or from public or private research centers.
L'archive ouverte pluridisciplinaire HAL, est destinée au dépôt et à la diffusion de documents scientifiques de niveau recherche, publiés ou non, émanant des établissements d'enseignement et de recherche français ou étrangers, des laboratoires publics ou privés. 


\title{
A trade-off between productivity and cost for the integrated part quality inspection and preventive maintenance planning under uncertainty
}

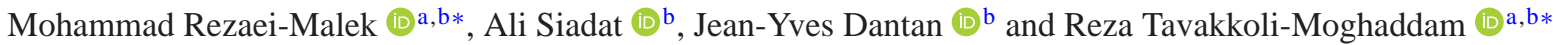 \\ ${ }^{a}$ School of Industrial Engineering, College of Engineering, University of Tehran, Tehran, Iran; ${ }^{b}$ Arts et Métiers ParisTech, LCFC,
} 57078 Metz, France

\begin{abstract}
This paper proposes a robust possibilistic and multi-objective mixed-integer linear programming mathematical model to concurrently plan part quality inspection and Preventive Maintenance (PM) activities for a serial multi-stage production system. This system contains the deteriorating stages and faces the uncertainty about estimated cost components and demand amount. The integrated model reaches two significant decisions which are the right time and place for performing the part quality inspection and PM. These decisions are made while the model is to simultaneously optimise the implied system productivity and total cost. To measure the implied system productivity, a new piecewise utility function for the ratio of produced conforming products to input workpieces is developed. A real case study and a numerical example are explored to validate and verify the developed model. The results prove the significance and effectiveness of considering the uncertainty and conflicting practical objectives for the problem.
\end{abstract}

Keywords: multi-stage production; preventive maintenance; inspection planning; productivity; uncertainty; multi-objective optimisation

\section{Introduction}

In Serial Multi-stage Manufacturing Systems (SMMSs), Part Quality Inspection Planning (PQIP) is a demanding problem, especially for the deteriorating ones. Since an SMMS presents the various possibilities for inspection, part quality inspection activities in the SMMS may be performed after some or every manufacturing stage (Mohammadi et al. 2018). Therefore, the PQIP problem includes many questions, such as when, where and in which extent inspection activities should be done along with a production process. Furthermore, determining appropriate defect management strategies (i.e. scrap, rework and repair/replacement) is required after detection of a defective item. It avoids the defect propagation all over the process and prevents defect shipment to consumer(s) (Rezaei-Malek et al. 2018a).

The PQIP problem has been explored by the scientists since the 1960s. The literature is wealthy on mathematical programming for the optimal allocation of inspection activities within the manufacturing system. Most of the early researches employed Dynamic Programming (DP) to handle the allocation problem in simple manufacturing systems free of inspection errors (e.g. see Lindsay and Bishop 1964; White 1966; Pruzan and Jackson 1967; White 1969). Lindsay and Bishop (1964) and White (1969) proved that an extreme point solution (either 0\% or 100\% inspection) is optimal for the linear cost functions and unconstrained systems. By developing an adaptive model, Pruzan and Jackson (1967) determined the optimal inspection strategy at a location regarding the inspection history. Eppen and Hurst (1974) extended the problem through considering two error types of inspection activities and applied the DP to solve the problem for an SMMS when all the rejected items considered to be scrapped. Yum and McDowellj (1987) proposed a Mixed-Integer Linear Programming (MILP) for the problem which could be solved by commercially available MILP packages such as GAMS.

Raz (1986), Mandroli, Shrivastava, and Ding (2006) and Shetwan, Vitanov, and Tjahjono (2011) reviewed the literature of PQIP problem in the different time periods. Recently, Rezaei-Malek et al. (2018b) have been updated the previous reviews and added new analyses about the incorporation of maintenance and production planning issues into the PQIP problem regarding the production quality paradigm developed by Colledani et al. (2014). Furthermore, they have investigated the multi-objective nature of the PQIP and the inherent uncertainty of the problem and the applied approaches for dealing with.

The three important aspects of each production system are production, quality, and maintenance that influence each other (Ben-Daya and Rahim 2001). There are some studies in the literature, whose paramount issue is the PQIP problem 
and have attempted to integrate this problem with the other production planning issues. In this respect, the integrated optimisation of inspection location with production planning (Gunter and Swanson 1985; Penn and Raviv 2007; Penn and Raviv 2008), required quantity of raw materials (Park, Park, and Ntuen 1988), batch size and reprocessing lot size for the rejected products (Tayi and Ballou 1988; Raghavachari and Tayi 1991; Raz, Herer, and Grosfeld-Nir 2000), cycle time and number of produced conforming items (Narahari and Khan 1996; Kakade, Valenzuela, and Smith 2004), timing and sequence of inspection activities (Kogan and Raz 2002), process planning (Shiau, Lin, and Chuang 2007), manufacturing shop scheduling and sequencing of inspection activities (Galante and Passannanti 2007; Sadegheih 2007), and production rate (Kim and Gershwin 2008) have been investigated.

Besides the production planning issues, different types of maintenance activity can be incorporated into the PQIP problem to improve the efficiency of production systems. For instance, Preventive Maintenance (PM) is used as a helpful tool for keeping the system in a good manufacturing state for manufacturing high-quality outcome. Defective Production Rate (DPR) as the principal input data for the PQIP is affected by the performance of PM activities, so there is an unassailable dependency of the PQIP on the PM plan. On the other side, the PM plan itself is specified based on the system deterioration trend and its status. To handle this dependency, as the first try, Rezaei-Malek et al. (2018c) incorporated the PM planning issue into the PQIP problem and proposed a programming mathematical model for an Integrated Part Quality Inspection and PM Planning (IPQIPMP) problem while satisfying the minimum demand amount. They assumed that each manufacturing stage deteriorates when time passes and accordingly the probability that a workpiece gets a defect is increasing. They determined the optimum time and place for performing part quality inspection and PM activities while minimising total manufacturing cost. They achieved a considerable cost saving in comparison with the separate optimisation of inspection and PM planning.

The IPQIPMP problem, like the other existing problems in manufacturing systems, inherently includes different sources of uncertainty. Some of these uncertainties are related to the internal reasons (e.g. Error type I and Error type II of inspection tools) and the others are rooted in the external reasons (e.g. cost fluctuations) (Rezaei-Malek et al. 2018b). In the PQIP literature, the first category of uncertainties was generally incorporated into the problem through a probabilistic approach (see e.g. Mohammadi et al. 2015) and the second one was considered by applying the fuzzy programming (see e.g. Azadeh et al. 2015b). Regarding the external-related uncertainties, the demand amount is a critical parameter that undergoes many changes because of the existence of cost fluctuations in the real market. However, there is no study in the PQIP and IPQIPMP literature handling the demand uncertainty. Indeed, this uncertain amount needs to be met by adjusting the system capacity through an appropriate planning of inspection and PM, and any shortage results in the loss of goodwill and back order penalty.

In the framework of the IPQIPMP problem, Rezaei-Malek et al. (2018c) just considered the internal reasons-related uncertainties through a probabilistic manner and ignored the uncertainties about the cost components and demand amount. Hence, this paper extends the proposed model by Rezaei-Malek et al. (2018c) and considers not only the internal reasonsrelated uncertainties but also the uncertainties about the cost components and demand amount and handles them through applying a Robust Possibilistic Programming (RPP) approach.

Probabilistic and fuzzy programming approaches are not capable to investigate the optimality and feasibility robustness of the obtained solutions. Indeed, it is significant to guarantee the obtained solution for being feasible and close to the optimal regarding different possible values of the uncertain parameters. This decreases the risk of SMMSs. Hence, this paper applies the RPP approach to guarantee the optimality and feasibility robustness of the obtained solution under the uncertainty of cost parameters and demand amount for the IPQIPMP problem.

Minimisation of the total cost consisting of production, inspection, repair/replacement, and scrap, is the most used form of the objective in the PQIP literature. On the other side, there is a lack of employing multi-objective models in the PQIP literature (Rezaei-Malek et al. 2018b). The PQIP problem inherently has a multi-objective nature where you must improve the quality of products by the resource assignment (e.g. inspection activities) and try to be profitable by reducing total cost. Mohammadi et al. (2018) is the only research work, which has proposed a bi-objective MILP model for the PQIP problem. The objective functions were the minimisation of total cost and the maximisation of customer satisfaction. They maximised customer satisfaction through minimising the penalty of delivered non-conforming items to the customer.

For the first time, this paper simultaneously considers two objective functions for the IPQIPMP problem. Besides the minimisation of the traditional total cost, a new objective function, called implied system productivity, is introduced to be maximised. Generally, a system productivity measure is expressed as the ratio of output to inputs used in a production process. Here, the output is equal to the number of produced conforming items. On the other side, typically, the implied utility of system productivity is considered equal to the obtained value for the ratio. However, regarding the studied case study of this paper, the implied utility may be different from the value of the ratio. For instance, based on our observation, the value 0.5 for the semiconductor producers is a catastrophe and for the oil pump housing manufacturers is a huge 
achievement. Accordingly, as the first objective function, this paper adopts a new piecewise utility function to reflect the case-based implied utility of the obtained system productivity ratio.

Briefly, this paper presents an MILP model for the IPQIPMP problem and contributes to the literature by:

- applying an RPP approach to deal with the uncertainty of cost components and demand amount in a robust way,

- introducing a new piecewise utility function to measure a case-based implied system productivity,

- presenting a bi-objective mathematical programming model for the IPQIPMP problem and obtaining the Paretooptimal solutions by the augmented $\varepsilon$-constraint approach.

The rest of this paper is organised as follows. The next section describes the problem and provides a bi-objective MILP model. The performed solution methods are elaborated in Section 3. Section 4 is dedicated to the computational experiment and a real case is explored. Section 5 draws the conclusions and provides some directions for future research.

\section{Problem description and mathematical model}

Consider a Serial $n$-Stage Manufacturing System (SnSMS). A single type product is produced by this discrete manufacturing process. Raw material enters the SnSMS, in which each production stage realises a certain Quality Characteristic (QC) on the product. All the workpieces cannot be processed in a perfect quality by the production stages. Because the production stages are not technologically capable to do their tasks without any fault. So, an item gets a defect after each stage with a certain probability. This probability is increasing over time (i.e. the manufacturing stages are deteriorating) and its value depends on the number of time periods, which has passed since the last performed PM. Therefore, at the beginning of each period, there is a chance to do a PM in an ambiguous cost on the different stages. In addition, after each stage, to avoid the defects propagation and defects shipment to end-customer(s), the system can perform an inspection activity in an imprecise cost. Each inspection activity can detect the defect happened at the preceding production stage. So, the defects propagation and accordingly energy and material wastage would be reduced. It is notable that Error type I and II exist for doing an inspection and a certain fraction of the rejected items can be repaired, and the rest should be scrapped while imposing ambiguous expenses to the SnSMS. Figure 1 shows the schematic plan of the considered SnSMS.

The SnSMS needs a simultaneous plan determining the right place and time for the PM and part quality inspection activities to manufacture conforming products. A planning horizon that includes equal time periods is considered for the SnSMS and the objective is to minimise total cost including production, inspection, repair, scrap, and PM and to maximise the implied system productivity. The cost components are not precisely certain and are represented by fuzzy rather than crisp values. To measure an implied system productivity, this research introduces a utility function of the obtained system productivity, $g(o)$, where $o$ is the proportion of the produced conforming products to the input workpieces. This function reflects that the implied system productivity is greater when it produces more conforming products with a fixed number of input workpieces (i.e. the $o$ proportion is closer to 1). The shape of this function may be different in dissimilar cases; however rationally, it is an increasing function. For instance, Figure 2(a) shows the function of implied system productivity in a production line for the oil pump housing, which is the considered case study of this paper. This function is an estimated base on the experts' opinions.

The problem assumptions are described as follows. Then, the needed notations are defined. At last, a Mixed-Integer Non-Linear Programming (MINLP) model is proposed for the above-mentioned problem.

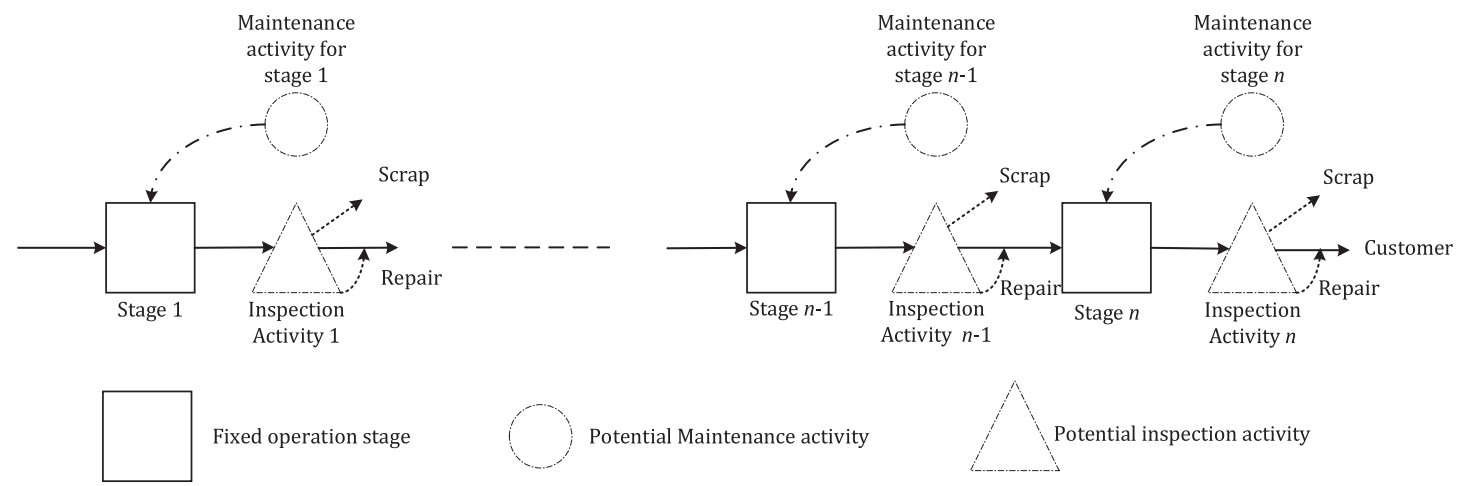

Figure 1. Schematic plan of the described IPQIPMP problem in each period. 


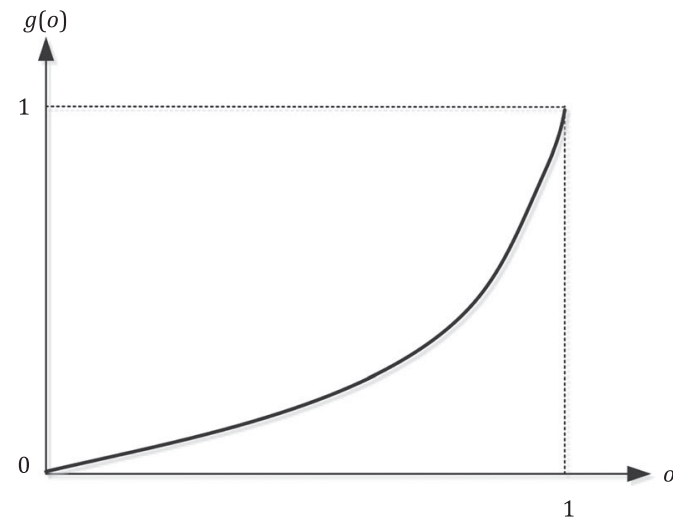

(a) Original shape of $g(0)$

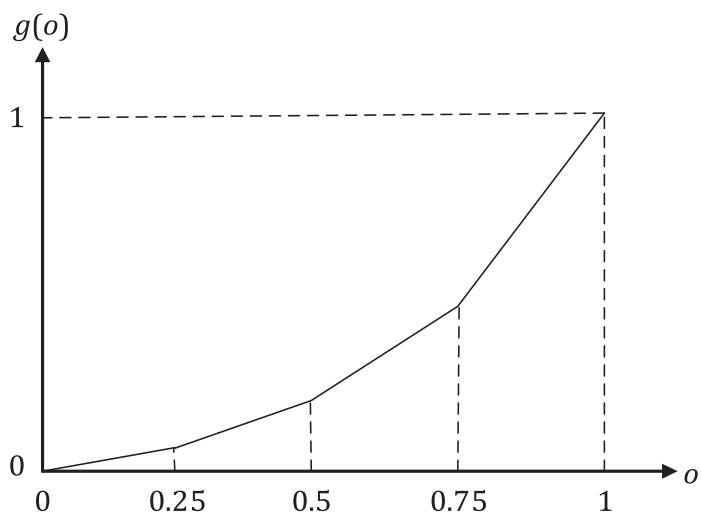

(b) Piecewise linear approximation of $g(o)$

Figure 2. Considered utility function for the fraction of the produced conforming products. (a) Original shape of $g(\mathrm{o})$. (b) Piecewise linear approximation of $g(o)$.

\subsection{Assumptions}

The principal assumptions of the described IPQIPMP problem are provided as follows:

- part defects are created only at the manufacturing stages,

- the values of Error type I and Error type II errors are known constant values during the planning horizon,

- the repair operation is performed perfectly and changes the rejected workpieces to the conforming ones.

\subsection{Indices}

$j \quad$ index of manufacturing stages $(j=1, \ldots, n)$

$t \quad$ index of time periods in the planning horizon $(t=1, \ldots, T)$

$i \quad$ index of passed time periods since the last done PM $(i=1, \ldots, T)$

\subsection{Parameters}

$n \quad$ number of manufacturing stages in the SnSMS

$\tilde{d e} \quad$ demand amount for conforming products in each period

$w_{t 01} \quad$ number of unit initial workpieces that enter the SnSMS in the period $t$

$\varepsilon_{i j} \quad$ probability that a workpiece gets a defect during processing in the manufacturing stage $j$ when $i$ time period has been passed since the last implemented PM on the manufacturing stage $j$

$\varepsilon_{t 0} \quad$ non-conforming fraction of initial workpieces that enters the SnSMS in the time period $t$

$\alpha_{j} \quad$ probability of the Error type I of the inspection activity $j$

$\beta_{j} \quad$ probability of the Error type II of the inspection activity $j$

$f_{j 1} \quad$ fraction of the rejected workpieces repaired at the inspection activity $j$

$f_{j 2} \quad$ fraction of the rejected workpieces scraped at the inspection activity $j$

$\widetilde{p}_{\dot{L}} \quad$ fuzzy unit production cost in the stage $j$

$i c_{j} \quad$ fuzzy unit inspection cost of the inspection activity $j$

$\widetilde{r c j 1}$

$\widetilde{r c_{j 2}}$

$\widetilde{s c_{j}}$

$\widetilde{m c_{j}}$

$\widetilde{p c} \quad$ fuzzy penalty cost of shipping a non-conforming workpiece to customer(s)

$y_{i} \quad$ auxiliary parameter that its value is equal to the number of its subscript (i.e. $i$ )

\subsection{Variables}

$d_{t j} \quad 1$; if inspection activity associated to the manufacturing stage $j$ is performed in the time period $t, 0$; otherwise 
$m_{t j} \quad 1$; if a PM activity corresponding to the production stage $j$ is done at the beginning of the time period $t$, 0 ; otherwise

$\omega_{t j} \quad$ in the time period $t$, a number of time periods that have been passed since the last implemented PM on the manufacturing stage $j$

$w_{t j 1} \quad$ expected number of conforming workpieces entering the manufacturing stage $j$ in the period $t$

$w_{t j 2} \quad$ expected number of non-conforming workpieces entering the manufacturing stage $j$ in the period $t$

$u_{t j 1} \quad=\alpha_{j}\left(1-\sum_{i=\omega^{L O}}^{\omega^{U P}} x_{i t j} \varepsilon_{i j}\right) w_{t j 1} d_{t j}$; expected number of conforming workpieces rejected by the $j$-th inspection activity opportunity in the time period $t$

$u_{t j 2}=\left(1-\beta_{j}\right)\left(\sum_{i=1}^{I} x_{i t j} \varepsilon_{i j} w_{t j 1}+w_{t j 2}\right) d_{t j}$; expected number of non-conforming workpieces rejected by the $j$-th inspection activity opportunity in the time period $t$

$x_{i t j} \quad$ auxiliary binary variable to activate appropriate $\varepsilon_{i j}$ parameter in the model

$\tilde{P} \quad$ fuzzy total production cost of the SnSMS

$\widetilde{I C} \quad$ fuzzy total part quality inspection cost of the SnSMS

$\widetilde{R C} \quad$ fuzzy total repair/replacement cost of the SnSMS

$\widetilde{M C} \quad$ fuzzy total PM cost of the SnSMS

$\widetilde{S C} \quad$ fuzzy total scrap cost of the SnSMS

$\widetilde{P C} \quad$ fuzzy total penalty cost of the SnSMS for delivering non-conforming items to customer(s)

\subsection{Bi-objective MINLP model}

Regarding the above-mentioned annotation, a bi-objective MINLP formulation of the IPQIPMP problem is developed as follows.

Model 1:

$$
\begin{aligned}
\operatorname{Max} z_{1} & =\sum_{t=1}^{T} g\left(\frac{w_{t, n+1,1}}{w_{t, 0,1}}\right) \\
\operatorname{Min} z_{2} & =R C+S C+P+I C+M C+P C
\end{aligned}
$$

s.t.

$$
\begin{gathered}
\widetilde{R C}=\sum_{t=1}^{T} \sum_{j=1}^{n} f_{j 1} \widetilde{r c_{j 1}} u_{t j 1}+\sum_{t=1}^{T} \sum_{j=1}^{n} f_{j 1} \widetilde{r c_{j 2}} u_{t j 2} \\
\widetilde{S C}=\sum_{t=1}^{T} \sum_{j=1}^{n} f_{j 2} \widetilde{s c_{j}} u_{t j 1}+\sum_{t=1}^{T} \sum_{j=1}^{n} f_{j 2} \widetilde{s c_{j}} u_{t j 2} \\
\widetilde{P C}=\sum_{t=1}^{T} \sum_{j=1}^{n} \widetilde{p}_{j}\left(w_{t j 1}+w_{t j 2}\right) \\
\widetilde{I C}=\sum_{t=1}^{T} \sum_{j=1}^{n} \widetilde{i c_{j}}\left(w_{t j 1}+w_{t j 2}\right) d_{t j} \\
\widetilde{M C}=\sum_{t=1}^{T} \sum_{j=1}^{n} \widetilde{m c_{j}} \times m d_{t j} \\
\widetilde{P C}=\sum_{t=1}^{T} \widetilde{p c} \times w_{t, n+1,2} \\
w_{t, 1,1}=\left(1-\widetilde{\varepsilon_{t 0}}\right) w_{t, 0,1} \quad \forall t
\end{gathered}
$$




$$
w_{t, 1,2}=\varepsilon_{t 0} w_{t 01} \forall t
$$

$$
\begin{aligned}
w_{t, j+1,1}= & \left(1-\sum_{i=1}^{I} x_{i t j} \varepsilon_{i j}\right) w_{t j 1} \\
& +\left(f_{j 1}\left(\alpha_{j}\left(1-\sum_{i=1}^{I} x_{i t j} \varepsilon_{i j}\right)+\left(1-\beta_{j}\right)\left(\sum_{i=1}^{I} x_{i t j} \varepsilon_{i j}\right)\right)-\alpha_{j}\left(1-\left(\sum_{i=1}^{I} x_{i t j} \varepsilon_{i j}\right)\right)\right) w_{t, j, 1} d_{t j} \\
& +f_{j 1}\left(1-\beta_{j}\right) w_{t j 2} d_{t j} \forall t, j \neq n \\
w_{t, n+1,1}= & \left(\begin{array}{l}
1- \\
+
\end{array}\right. \\
+ & \left.\left.\left(\sum_{i=1}^{I} x_{i t n} \varepsilon_{i n}\right)\right) w_{t n 1}\left(\alpha_{n}\left(1-\left(\sum_{i=1}^{I} x_{i t n} \varepsilon_{i n}\right)\right)+\left(1-\beta_{n}\right)\left(\sum_{i=1}^{I} x_{i t n} \varepsilon_{i n}\right)\right)-\alpha_{n}\left(1-\left(\sum_{i=1}^{I} x_{i t n} \varepsilon_{i n}\right)\right)\right) w_{t, n, 1} d_{t n} \\
& +f_{n 1}\left(1-\beta_{n}\right) w_{t n 2} d_{t n} \forall t
\end{aligned}
$$

$$
\begin{gathered}
w_{t, j+1,2}=w_{t j 2}+\left(\sum_{i=1}^{I} x_{i t j} \varepsilon_{i j}\right) w_{t j 1}-\left(1-\beta_{j}\right)\left(\sum_{i=1}^{I} x_{i t j} \varepsilon_{i j}\right) w_{t j 1} d_{t j} \\
-\left(1-\beta_{j}\right) w_{t j 2} d_{t j} \quad \forall t, j \\
\omega_{t j}=\left(\omega_{t-1, j}+1\right)\left(1-m d_{t j}\right) \quad \forall j, t \neq 1 \\
\omega_{1, j}=0 \quad \forall j \\
m d_{1 j}=1 \quad \forall j \\
\omega_{t j}=\sum_{i=1}^{T} x_{t j i} y_{i} \quad \forall t, j \\
\sum_{i=1}^{T} x_{t j i}=1 \quad \forall t, j \\
w_{t, n+1,1} \geq \tilde{d e} \quad \forall t
\end{gathered}
$$

$$
w_{t j 1}, w_{t j 2}, u_{t j 1},, u_{t j 2}, R C, I C,, P, M C, S C, P C \geq 0 \quad \forall t, j
$$

$$
m d_{t j}, d_{t j} \text { : binary decisions } \forall t, j ; x_{i t j} \text { : binary } \forall t, j, i ; \omega_{t j}: \text { positive integer } \forall t, j
$$

Objective function (1) maximises the implied system productivity through maximising the utility function of the produced conforming products to the input workpieces. As mentioned before, the traditional measure for the system productivity was the proportion of output (number of produced conforming items) to input (number of initial workpieces); however, the implied system productivity is different in dissimilar cases and it is possible to reflect these different implications through considering various functions. For example, Figure 2(a) shows the implied system productivity for the considered case study in Section 4.2. Hereafter this proportion is called as $o_{t}$ variable. Objective (2) minimises the total cost including repair, scrap, production, inspection, PM, and penalty cost. These two objectives can be handled by a multi-objective optimisation programming method (please see Section 3.3) to obtain a group of non-dominated solutions. Equations (3)-(8) calculate the different cost components. Equations (9)-(13) obtain the expected number of conforming and non-conforming parts entering the different manufacturing stages in each time period. Equation (14) obtains the number of passed periods after the last implemented PM. It is assumed that a PM is performed for all the manufacturing stages in the first period (see Equations (15) and (16)). Equations (17) and (18) activate the corresponding auxiliary binary variable $x_{i t j}$ to $\varepsilon_{i j}$ and accordingly the 
term $\left(\sum_{i=1}^{T} x_{i j} \varepsilon_{i j}\right)$ in each period $t$ take the value of corresponding $\varepsilon_{i j}$ regarding the number of the passed period since the last performed PM. Constraint (19) imposes the SMMS to satisfy the demand of customer(s) in each time period. Equation (20) is a non-negativity restriction, and Equation (21) shows that performing an inspection activity and performing a PM are binary decisions. In addition, Equation (21) shows that the $x_{i t j}$ auxiliary binary variable and the $\omega_{t j}$ are integer positive variables.

\section{Methodology}

To Deal with the non-linearity, multi-objectivity, and uncertainty of some parameters of the Model 1, a three-step approach is developed. Figure 3 depicts the proposed approach and the explanation of each step is provided in the following sub-sections.

\subsection{Step 1: linearisation procedure}

Model 1 is a bi-objective MINLP model and we try to linearise Model 1 through some conventional operations research techniques. To this aim, new variables and constraints are added to Model 1 for the linearisation purpose. The multiplications of two real variables (i.e. $w_{t j 1} \times d_{t j}, w_{t j 2} \times d_{t j}, x_{i t j} \times w d_{t j 1}, x_{i t j} \times w d_{t j 2}, x_{i t j} \times w_{t j 1}$ ) are linearised by replacing them with new real variables $\left(w d_{t j 1}, w d_{t j 2}, x w d_{t j 1}, x w d_{t j 2}, x w_{t j 1}\right)$ and adding new constraints (i.e. Equations (34)-(36), (37)-(39), (42)(44), (45)-(47), (48)-(50)). In addition, the multiplication of positive integer $\omega_{t-1, j}$ and binary $m d_{t j}$ is replaced with new real variable $\omega m d_{t-1, t, j}$ and adding Constraints (40) and (41).

After the above-mentioned linearisation procedure, Model 1 is still non-linear because of the first objective function. This non-linearity can be handled by employing a piecewise linear approximation to this non-linear function which is shown in Figure 2(b). The curve in Figure 2(a) is divided into four straight line segments and is formulated as follows:

$$
g(o)= \begin{cases}\frac{4 o}{13} & o<0.25 \\ \frac{8 o-1}{13} & 0.25 \leq o<0.5 \\ \frac{16 o-5}{13} & 0.5 \leq o<0.75 \\ \frac{24 o-11}{13} & o \geq 0.25\end{cases}
$$

Applying more portions to approximate the curve results in more accuracy; however, as explained in Section 4.2, four portions obtains the satisfying accuracy for the Decision-Maker (DM) of the considered case study. The aim is to remove the non-linear term $g\left(w_{t, n+1,1} / w_{t, 1,1}\right)$ from Model 1 . This can be performed by replacing it through the single linear term $\vartheta_{t}$. Then, it is possible to relate $\vartheta_{t}$ to $o_{t}$ by the following formulations.

$$
\begin{gathered}
o_{t}-0 \times \lambda 1_{t}+\frac{1}{4} \times \lambda 2_{t}+\frac{1}{2} \times \lambda 3_{t}+\frac{3}{4} \times \lambda 4_{t}+1 \times \lambda 5_{t}=0 \quad \forall t \\
\vartheta_{t}-0 \times \lambda 1_{t}+\frac{1}{13} \times \lambda 2_{t}+\frac{3}{13} \times \lambda 3_{t}+\frac{7}{13} \times \lambda 4_{t}+1 \times \lambda 5_{t}=0 \quad \forall t \\
\lambda 1_{t}+\lambda 2_{t}+\lambda 3_{t}+\lambda 4_{t}+\lambda 5_{t}=0 \quad \forall t
\end{gathered}
$$

Some new variables are added into Model 1 (i.e. $\lambda v$ ). These can be implied as 'weights' to be attached to the vertices of the curve in Figure 2(b). In addition, it is necessary to consider another constraint regarding $\lambda v$.

At most two adjacent $\lambda v$ can be non-zero

Constraint (24) can be done by introducing $\lambda v$ under SOS2 condition. Stipulation (24) guarantees that corresponding values of $\vartheta_{t}$ and $o_{t}$ lie on one of the straight line portions. For example, if $\lambda 2=0.5$ and $\lambda 3=0.5$ (i.e. other $\lambda v$ are zero), we can

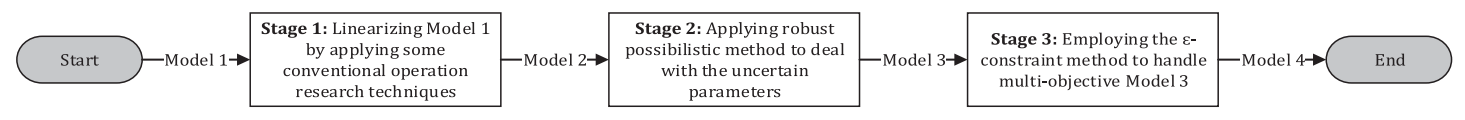

Figure 3. Flowchart of the proposed three-stage approach. 
get $o_{t}=0.375$ and $\vartheta_{t}=0.154$. Apparently, neglecting Constraint (24) will erroneously let the possibility of values $o_{t}$ and $\vartheta_{t}$ off the piecewise straight lines. For further information about the piecewise approximation approach, enthusiastic readers can refer to Williams (2013).

By implementing the afore-mentioned linearisation operation, Model 1 is changed to the linear Model 2 as follows:

Model 2:

$$
\operatorname{Max} z_{1}=\sum_{t=1}^{T} \vartheta_{t}
$$

$$
\operatorname{Min} z_{2}=\widetilde{R C}+\widetilde{S C}+\tilde{P}+\widetilde{I C}+\widetilde{M C}+\widetilde{P C}
$$

s.t.

$$
\begin{aligned}
& \widetilde{R C}=\sum_{t=1}^{T} \sum_{j=1}^{n} f_{j 1} \widetilde{r c_{j 1}} \alpha_{j} w d_{t j 1}-f_{j 1} \widetilde{r c_{j 1}} \alpha_{j}\left(\sum_{i=1}^{T} \varepsilon_{i j} x w d_{t j i 1}\right) \\
& +\sum_{t=1}^{T} \sum_{j=1}^{n} f_{j 1} \widetilde{r c_{j 2}}\left(\sum_{i=1}^{T} \varepsilon_{i j} x w d_{t j i 1}\right)+f_{j 1} \widetilde{r c_{j 2}} w d_{t j 2} \\
& -f_{j 1} \widetilde{r c_{j 2}} \beta_{j}\left(\sum_{i=1}^{T} \varepsilon_{i j} x w d_{t j i 1}\right)-f_{j 1} \widetilde{r c_{j 2}} \beta_{j} w d_{t j 2} \\
& \widetilde{S C}=\sum_{t=1}^{T} \sum_{j=1}^{n} f_{j 2} \widetilde{S c}_{j} \alpha_{j} w d_{t j 1}-f_{j 2} \widetilde{S c}_{j} \alpha_{j}\left(\sum_{i=1}^{T} \varepsilon_{i j} x w d_{t j i 1}\right) \\
& +\sum_{t=1}^{T} \sum_{j=1}^{n} f_{j 2} \widetilde{s c_{j}}\left(\sum_{i=1}^{T} \varepsilon_{i j} x w d_{t j i 1}\right)+f_{j 2} \widetilde{s c}_{j} w d_{t j 2}-f_{j 2} \widetilde{s c_{j}} \beta_{j}\left(\sum_{i=1}^{T} \varepsilon_{i j} x w d_{t j i 1}\right) \\
& -f_{j 2} \widetilde{s c}_{j} \beta_{j} w d_{t j 2} \\
& w_{t, j+1,1}=w_{t j 1}-\left(\sum_{i=1}^{T} \varepsilon_{i j} x w_{t j i 1}\right)+f_{j 1} \alpha_{j} w d_{t j 1}-f_{j 1} \alpha_{j}\left(\sum_{i=1}^{T} \varepsilon_{i j} x w d_{t j i 1}\right)+f_{j 1}\left(\sum_{i=1}^{T} \varepsilon_{i j} x w d_{t j i 1}\right) \\
& -f_{j 1} \beta_{j}\left(\sum_{i=1}^{T} \varepsilon_{i j} x w d_{t j i 1}\right)-\alpha_{j} w d_{t j 1}-\alpha_{j}\left(\sum_{i=1}^{T} \varepsilon_{i j} x w d_{t j i 1}\right)+f_{j 1} w d_{t j 2} \\
& -f_{j 1} \beta_{j} w d_{t j 2} \quad \forall t, j \neq n \\
& w_{t, n+1,1}=w_{t n 1}-\left(\sum_{i=1}^{T} \varepsilon_{i n} x w_{t n i 1}\right)+f_{n 1} \alpha_{n} w d_{t n 1}-f_{n 1} \alpha_{n}\left(\sum_{i=1}^{T} \varepsilon_{i n} x w d_{t n i 1}\right) \\
& +f_{n 1}\left(\sum_{i=1}^{T} \varepsilon_{i n} x w d_{t n i 1}\right)-f_{n 1} \beta_{n}\left(\sum_{i=1}^{T} \varepsilon_{i n} x w d_{t n i 1}\right)-\alpha_{n} w d_{t n 1} \\
& -\alpha_{n}\left(\sum_{i=1}^{T} \varepsilon_{i n} x w d_{t n i 1}\right)+f_{n 1} w d_{t n 2}-f_{n 1} \beta_{n} w d_{t n 2} \quad \forall t \\
& w_{t, j+1,2}=w_{t j 2}+\left(\sum_{i=1}^{T} \varepsilon_{i n} x w_{t j i 1}\right)-\left(\sum_{i=1}^{T} \varepsilon_{i j} x w d_{t j i 1}\right)+\beta_{j}\left(\sum_{i=1}^{T} \varepsilon_{i j} x w d_{t j i 1}\right)-w d_{t j 2}+\beta_{j} w d_{t j 2} \quad \forall t, j \\
& \omega_{t j}=\omega_{t-1, j}+1-\omega m d_{t-1, t, j}+m d_{t j} \quad \forall j, t \neq 1
\end{aligned}
$$




$$
\begin{aligned}
& w d_{t j 1} \leq M \times d_{t j} \forall t, j \\
& w d_{t j 1} \leq w_{t j 1} \forall t, j \\
& w d_{t j 1} \geq w_{t j 1}-\left(1-d_{t j}\right) \times M \forall t, j w d_{t j 2} \leq \\
& M \times d_{t j} \forall t, j \\
& w d_{t j 2} \leq w_{t j 2} \forall t, j \\
& w d_{t j 2} \geq w_{t j 2}-\left(1-d_{t j}\right) \times M \forall t, j \\
& \omega_{t j}^{L O} \times m d_{t j} \leq \omega m d_{t-1, t, j} \leq \omega_{t j}^{U P} \times m d_{t j} \quad \forall j, t \neq 1 \\
& \omega_{t-1, j}-\omega_{t j}^{U P} \times\left(1-m d_{t j}\right) \leq \omega m d_{t-1, t, j} \leq \omega_{t-1, j}-\omega_{t j}^{L O} \times\left(1-m d_{t j}\right) \quad \forall j, t \neq 1 \\
& w d_{i t j 1} \leq M \times x_{i t j} \quad \forall t, j \\
& x w d_{i t j 1} \leq w d_{t j 1} \quad \forall t, j \\
& x w d_{i t j 1} \geq w d_{t j 1}-\left(1-x_{i t j}\right) \times M \quad \forall i, t, j \\
& x w d_{i t j 2} \leq M \times x_{i t j} \quad \forall i, t, j \\
& x w d_{i t j 2} \leq w d_{t j 2} \quad \forall i, t, j \\
& x w d_{i t j 2} \geq w d_{t j 2}-\left(1-x_{i t j}\right) \times M \quad \forall i, t, j \\
& x w_{i t j 1} \leq M \times x_{i t j} \quad \forall i, t, j \\
& x w_{i t j 1} \leq w_{t j 1} \quad \forall i, t, j \\
& x w_{i t j 1} \geq w_{t j 1}-\left(1-x_{i t j}\right) \times M \quad \forall i, t, j \\
& w d_{t j 1}, w d_{t j 2}, x w d_{i t j 1}, x w d_{i t j 2}, x w_{i t j 1} \geq 0 \quad \forall t, j ; \omega m d_{t-1, t, j} \geq 0 \quad \forall j, t \neq 1 \\
& \lambda v: \text { Positive and SOS2 } v \in\{1, \ldots, 5\}
\end{aligned}
$$

With Equations (5)-(10) and (15)-(24).

\subsection{Step 2: robust possibilistic programming (RPP)}

As can be implied from the related literature, cost components and demand amount of the PQIP problem face a high degree of uncertainty in a real-life condition since the dynamic nature of the market and the tactical horizon of planning decisions. Specifically, when external reasons are considered, the degree of uncertainty increased notably. So, ignoring the uncertainty in the planning of the manufacturing system may pose high risks to the company. Examples of the risks are a considerable amount of back order and misestimated manufacturing cost. To deal with these uncertain parameters that are based on the subjective opinion of the DM(s) and to obtain a robust solution, this paper employs the Robust Possibilistic Approach (RPP) developed by Pishvaee, Razmi, and Torabi (2012).

Fuzzy mathematical programming is classified into two major categories (Pishvaee, Razmi, and Torabi 2012; Zahiri, Tavakkoli-Moghaddam, and Rezaei-Malek 2016): possibilistic programming and flexible programming. Possibilistic programming handles vague coefficients of constraints and objective functions that are generally formulated considering available objective data and subjective knowledge of DM. Flexible programming is used to handle flexible target value of goals and limitations (Pishvaee, Razmi, and Torabi 2012). With the above-mentioned definitions, Model 2 belongs to the possibilistic programming category. 


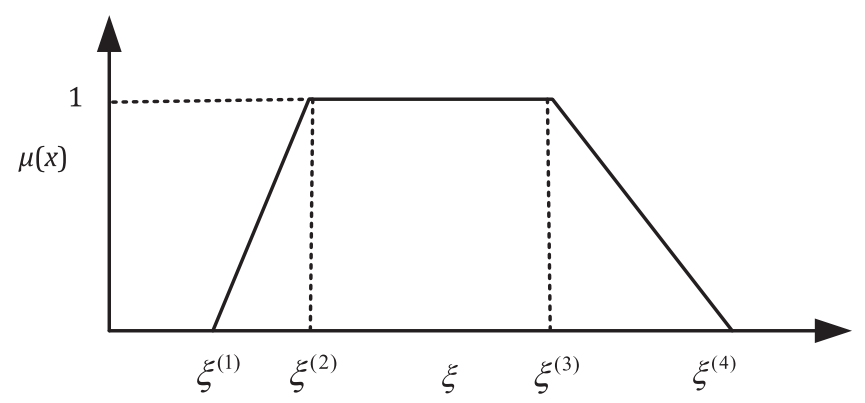

Figure 4. Possibility distribution of trapezoidal fuzzy number $\tilde{\xi}$.

Pishvaee, Razmi, and Torabi (2012) presented a new approach, namely RPP. They extended the theory of robust programming into the possibilistic programming framework. This approach has the capabilities of both possibilistic programming and the concept of robustness. In comparison with the basic possibilistic chance-constrained programming, the RPP approach prevents subjective judgment about the best value of chance constraints' confidence levels (i.e. $\theta$ ) as well as guaranteeing to obtain the global optimum value for them. This advantage is more valuable when the number of chance constraints rises and there is no need to apply complex and time-consuming processes like simulation experiments to obtain the optimum value for confidence levels. Generally, the RPP model searches for a reasonable trade-off between (1) feasibility robustness, (2) optimality robustness and (3) average performance. Several versions of the RPP approach was introduced by Pishvaee, Razmi, and Torabi (2012). Because in our application it is advantageous for the DMs to obtain a lower total cost when compared to the expected optimal value in any realisation. So, in this case, the Robust Possibilistic Programming-II (RPP-II) version is applied. For more information about the other versions of the RPP programming approach, enthusiastic readers can refer to Pishvaee, Razmi, and Torabi (2012). Here, according to the available data type in the considered case study in Section 4.2, trapezoidal possibility distributions (see Figure 4) are adapted to model the imprecise parameters. Trapezoidal fuzzy numbers can be represented by their four prominent points (e.g. $\left.\tilde{\xi}=\left(\xi^{(1)}, \xi^{(2)}, \xi^{(3)}, \xi^{(4)}\right)\right)$.

Model 3:

This model is completely same as Model 2 except the second objective function and Constraint (19) which are changed based on the RPP-II as follows:

$$
\operatorname{Min} E\left[z_{2}\right]+\gamma\left(z_{2}^{\max }-E\left[z_{2}\right]\right)+\delta\left[d e^{(4)}-(1-\theta) d e^{(3)}-\theta d e^{(4)}\right]
$$

s.t.

$$
w_{t, n+1,1} \geq(1-\theta) d e^{(3)}+\theta d e^{(4)} \quad \forall t
$$

where:

$$
\begin{aligned}
E\left[z_{2}\right] & =E[R C]+E[S C]+E[P]+E[I C]+E[M C]+E[P C] \\
z_{2}^{\max }= & R C^{\max }+S C^{\max }+P^{\max }+I C^{\max }+M C^{\max }+P C^{\max } \\
E[R C]= & \sum_{t=1}^{T} \sum_{j=1}^{n} f_{j 1}\left(\frac{r c_{j 1}^{(1)}+r c_{j 1}^{(2)}+r c_{j 1}^{(3)}+r c_{j 1}^{(4)}}{4}\right) \alpha j w d_{t j 1} \\
& -f_{j 1}\left(\frac{r c_{j 1}^{(1)}+r c_{j 1}^{(2)}+r c_{j 1}^{(3)}+r c_{j 1}^{(4)}}{4}\right) \alpha j\left(\sum_{i=1}^{T} \varepsilon_{i j} x w d_{t j i 1}\right) \\
& +\sum_{t=1}^{T} \sum_{j=1}^{n} f_{j 1}\left(\frac{r c_{j 2}^{(1)}+r c_{j 2}^{(2)}+r c_{j 2}^{(3)}+r c_{j 2}^{(4)}}{4}\right)\left(\sum_{i=1}^{T} \varepsilon_{i j} x w d_{t j i 1}\right)
\end{aligned}
$$




$$
\begin{aligned}
& +f_{j 1}\left(\frac{r c_{j 2}^{(1)}+r c_{j 2}^{(2)}+r c_{j 2}^{(3)}+r c_{j 2}^{(4)}}{4}\right) w d_{t j 2} \\
& -f_{j 1}\left(\frac{r c_{j 2}^{(1)}+r c_{j 2}^{(2)}+r c_{j 2}^{(3)}+r c_{j 2}^{(4)}}{4}\right) \beta_{j}\left(\sum_{i=1}^{T} \varepsilon_{i j} x w d_{t j i 1}\right) \\
& -f_{j 1}\left(\frac{r c_{j 2}^{(1)}+r c_{j 2}^{(2)}+r c_{j 2}^{(3)}+r c_{j 2}^{(4)}}{4}\right) \beta_{j} w d_{t j 2} \\
& R C^{\max }=\sum_{t=1}^{T} \sum_{j=1}^{n} f_{j 1} r c_{j 1}^{(4)} \alpha j w d_{t j 1}-f_{j 1} r c_{j 1}^{(4)} \alpha j\left(\sum_{i=1}^{T} \varepsilon_{i j} x w d_{t j i 1}\right) \\
& +\sum_{t=1}^{T} \sum_{j=1}^{n} f_{j 1} r c_{j 2}^{(4)}\left(\sum_{i=1}^{T} \varepsilon_{i j} x w d_{t j i 1}\right)+f_{j 1} r c_{j 2}^{(4)} w d_{t j 2} \\
& -f_{j 1} r c_{j 2}^{(4)} \beta_{j}\left(\sum_{i=1}^{T} \varepsilon_{i j} x w d_{t j i 1}\right)-f_{j 1} r c_{j 2}^{(4)} \beta_{j} w d_{t j 2} \\
& E[S C]=\sum_{t=1}^{T} \sum_{j=1}^{n} f_{j 2}\left(\frac{s c_{j}^{(1)}+s c_{j}^{(2)}+s c_{j}^{(3)}+s c_{j}^{(4)}}{4}\right) \alpha_{j} w d_{t j 1} \\
& -f_{j 2}\left(\frac{s c_{j}^{(1)}+s c_{j}^{(2)}+s c_{j}^{(3)}+s c_{j}^{(4)}}{4}\right) \alpha_{j}\left(\sum_{i=1}^{T} \varepsilon_{i j} x w d_{t j i 1}\right) \\
& +\sum_{t=1}^{T} \sum_{j=1}^{n} f_{j 2}\left(\frac{s c_{j}^{(1)}+s c_{j}^{(2)}+s c_{j}^{(3)}+s c_{j}^{(4)}}{4}\right)\left(\sum_{i=1}^{T} \varepsilon_{i j} x w d_{t j i 1}\right) \\
& +f_{j 2}\left(\frac{s c_{j}^{(1)}+s c_{j}^{(2)}+s c_{j}^{(3)}+s c_{j}^{(4)}}{4}\right) w d_{t j 2} \\
& -f_{j 2}\left(\frac{s c_{j}^{(1)}+s c_{j}^{(2)}+s c_{j}^{(3)}+s c_{j}^{(4)}}{4}\right) \beta_{j}\left(\sum_{i=1}^{T} \varepsilon_{i j} x w d_{t j i 1}\right) \\
& -f_{j 2}\left(\frac{s c_{j}^{(1)}+s c_{j}^{(2)}+s c_{j}^{(3)}+s c_{j}^{(4)}}{4}\right) \beta_{j} w d_{t j 2} \\
& S C^{\max }=\sum_{t=1}^{T} \sum_{j=1}^{n} f_{j 2} s c_{j}^{(4)} \alpha_{j} w d_{t j 1}-f_{j 2} s c_{j}^{(4)} \alpha_{j}\left(\sum_{i=1}^{T} \varepsilon_{i j} x w d_{t j i 1}\right) \\
& +\sum_{t=1}^{T} \sum_{j=1}^{n} f_{j 2} s c_{j}^{(4)}\left(\sum_{i=1}^{T} \varepsilon_{i j} x w d_{t j i 1}\right)+f_{j 2}\left(\frac{s c_{j}^{(1)}+s c_{j}^{(2)}+s c_{j}^{(3)}+s c_{j}^{(4)}}{4}\right) w d_{t j 2} \\
& -f_{j 2} s c_{j}^{(4)} \beta_{j}\left(\sum_{i=1}^{T} \varepsilon_{i j} x w d_{t j i 1}\right)-f_{j 2} s c_{j}^{(4)} \beta_{j} w d_{t j 2} \\
& E[P]=\sum_{t=1}^{T} \sum_{j=1}^{n}\left(\frac{p_{j}^{(1)}+p_{j}^{(2)}+p_{j}^{(3)}+p_{j}^{(4)}}{4}\right)\left(w_{t j 1}+w_{t j 2}\right) \\
& P^{\max }=\sum_{t=1}^{T} \sum_{j=1}^{n} p_{j}^{(4)}\left(w_{t j 1}+w_{t j 2}\right)
\end{aligned}
$$




$$
\begin{gathered}
E[I C]=\sum_{t=1}^{T} \sum_{j=1}^{n}\left(\frac{i c_{j}^{(1)}+i c_{j}^{(2)}+i c_{j}^{(3)}+i c_{j}^{(4)}}{4}\right)\left(w_{t j 1}+w_{t j 2}\right) d_{t j} \\
I C^{\max }=\sum_{t=1}^{T} \sum_{j=1}^{n} i c_{j}^{(4)}\left(w_{t j 1}+w_{t j 2}\right) d_{t j} \\
E[M C]=\sum_{t=1}^{T} \sum_{j=1}^{n}\left(\frac{m c_{j}^{(1)}+m c_{j}^{(2)}+m c_{j}^{(3)}+m c_{j}^{(4)}}{4}\right) \times m d_{t j} \\
M C^{\max }=\sum_{t=1}^{T} \sum_{j=1}^{n} m c_{j}^{(4)} \times m d_{t j} \\
E[P C]=\sum_{t=1}^{T}\left(\frac{p c_{j}^{(1)}+p c_{j}^{(2)}+p c_{j}^{(3)}+p c_{j}^{(4)}}{4}\right) \times w_{t, n+1,2} \\
P C^{\max }=\sum_{t=1}^{T} p c_{j}^{(4)} \times w_{t, n+1,2}
\end{gathered}
$$

The significance of the second term against the other terms in the function is represented by $\gamma$ and this term controls optimality robustness. The third term specifies the confidence level of each chance constraint where $\delta$ is the penalty unit of possible violation of each constraint containing vague parameter(s) and $\left[d e^{(4)}-(1-\theta) d e^{(3)}-\theta d e^{(4)}\right]$ shows the difference between the worst-case value of vague demand parameter and the value that is applied in chance constraints. Hence, the feasibility robustness of the solution vector is controlled by this term. Noteworthy, that is not just a meaningless and theoretical parameter, rather the value of penalty can be properly specified regarding the application context (Pishvaee, Razmi, and Torabi 2012).

\subsection{Step 3: single objective counterpart Model}

In this paper, the augmented $\varepsilon$-constraint technique is used to transform the bi-objective Model 3 to the single objective counterpart. First, the traditional $\varepsilon$-constraint technique is explored and then its augmented version is elaborated. In the general $\varepsilon$-constraint technique, the most significant objective function (i.e. the first objective in this research that is chosen regarding the opinion of the DM) is optimised while the other objective (i.e. the second function) is transformed into a constraint as follows.

$$
\operatorname{Max} z_{1}=\sum_{t=1}^{T} \vartheta_{t}
$$

s.t.

$$
E\left[z_{2}\right]+\gamma\left(z_{2}^{\max }-E\left[z_{2}\right]\right)+\delta\left[d e^{(4)}-(1-\theta) d e^{(3)}-\theta d e^{(4)}\right] \leq \varepsilon_{2}
$$

Equations (15)-(24), (30)-(52) and (54)-(68).

Then, the efficient solutions of Model 4 are achieved by parametrical variation in the RHS (i.e. $\varepsilon_{2}$ ) of the constrained objective function (Mavrotas 2009). The range of $\varepsilon_{2}$ can be obtained by optimising the constrained second objective functions separately considering the constraints and constructing the payoff table (Rezaei-Malek et al. 2016). Next, different values for $\varepsilon_{2}$ can be obtained by dividing the range of the constrained second objective (i.e. $r_{2}$ ) to $q$ equal intervals as follows.

$$
r_{2}=z_{2}^{\max }-z_{2}^{\min } ; \varepsilon_{2}^{l}=z_{2}^{\max }-\frac{r_{2}}{q} \times l \quad l=0, \ldots, q-1
$$

The traditional form of the $\varepsilon$-constraint method has some drawbacks. For example, this method does not guarantee the efficiency of the achieved solutions (i.e. achieving weakly efficient solutions) (Rossit et al. 2017). Mavrotas (2009) explained 
some of these drawbacks and proposed an improved version of the $\varepsilon$-constraint technique, called 'augmented $\varepsilon$-constraint method'. The formulation of the augmented $\varepsilon$-constraint technique for the Model 3 is as follows.

Model 4:

$$
\operatorname{Max} z_{1}+\left(\varphi_{2} \times s_{2}\right)
$$

s.t.

$$
E\left[z_{2}\right]+\gamma\left(z_{2}^{\max }-E\left[z_{2}\right]\right)+s_{2}=\varepsilon_{2}
$$

Equations(15)-(23), (29)-(51) and (53)-(66)

$$
s_{2}>0
$$

where $\varphi_{2}$ is a sufficiently small number (generally between $10^{-3}$ and $10^{-6}$ ) and augmented term $\varphi_{2} \times s_{2}$ guarantees achieving only an efficient solution for each epsilon vector. This approach is then used to solve the bi-objective model to obtain efficient solutions.

\section{Experimental results}

\subsection{Model verification}

To verify the presented implied system productivity objective function and the RPP-II approach, a numerical example is explored. This numerical example and its features specifications are inspired from a part of a real SMMS that is an assembly line for 'PC250' reciprocating compressor. It contains a three-stage system and the duration of the planning horizon is considered 12 periods. All the three stages have the same deterioration processes because the system contains identical machines for performing different operations. According to the historical data, the $\varepsilon_{i j}$ behaviour of each manufacturing stage as a function of $i$ is estimated as $\varepsilon_{i j}=0.05 \times i$. The number of the unit material that enters the SMMS is 100 units per time period and $5 \%$ of them are non-conforming items (i.e. $\varepsilon_{t 0}=0.05$ ). Uniform distribution is used to randomly generate prominent values of each trapezoidal fuzzy number while the ranges are estimated according to the available data and experts' knowledge of the assembly line for 'PC250' (see Table 1). Similarly, the value of $\gamma$ and $\delta$ are experimentally selected 0.2 and 100 , respectively.

The presented models are coded in the GAMS software (ver. 24.1.2) and handled through the CPLEX solver (ver. 12.5.1.0), that is capable to solve MILP models by the branch-and-cut algorithm, on the data of the case study and the numerical example applying a laptop with Intel ${ }^{\circledast}$ Core $^{\mathrm{TM}}$ i7-6300U CPU, $1.73 \mathrm{GHz}$ and $8 \mathrm{~GB}$ of RAM and Windows7 operating system is applied as a technical platform.

Table 2 depicts the generated solutions using different objective functions of the proposed Model 3. Since the combination of the performed PM and inspection activities in each solution are different, it can be implied that the results from employing different objective functions are not necessarily consistent, and so objective functions should be considered separately. In addition, Table 3 shows the detail of the obtained solution by the deterministic Model 2 (considering the single objective function (27)) and the right part of Table 2 illustrates the solution of the uncertain Model 3 (considering the single objective function (53)). The difference between two solutions proves the influence of the RPP-II method on the optimum obtained solution.

Figure 5 depicts the values of the objective functions of the Pareto-optimal solutions obtained by solving Model 4. This observation also proves the above-mentioned conclusion as an increase of the implied system productivity (i.e. the first

Table 1. Data of the numerical example.

\begin{tabular}{ll}
\hline Uncertain parameter & \multicolumn{1}{c}{ Distribution } \\
\hline$\widetilde{p_{j}}=\left(p_{j}^{(1)}, p_{j}^{(2)}, p_{j}^{(3)}, p_{j}^{(4)}\right)$ & $\sim \mathrm{U}(1,50) ; p_{j}^{(i+1)}>p_{j}^{(i)}$ \\
$\tilde{i c_{j}}=\left(i c_{j}^{(1)}, i c_{j}^{(2)}, i c_{j}^{(3)}, i c_{j}^{(4)}\right)$ & $\sim \mathrm{U}(0,2) ; i c_{j}^{(i+1)}>i c_{j}^{(i)}$ \\
$\widetilde{r c_{j 1}}=\left(r c_{j 1}^{(1)}, r c_{j 1}^{(2)}, r c_{j 1}^{(3)}, r c_{j 1}^{(4)}\right)$ & $\sim \mathrm{U}(1,10) ; r c_{j 1}^{(i+1)}>r c_{j 1}^{(i)}$ \\
$\widetilde{r c_{j 2}}=\left(r c_{j 2}^{(1)}, r c_{j 2}^{(2)}, r c_{j 2}^{(3)}, r c_{j 2}^{(4)}\right)$ & $\sim \mathrm{U}(1,50) ; r c_{j 2}^{(i+1)}>r c_{j 2}^{(i)}$ \\
$\widetilde{s c_{j}}=\left(s c_{j}^{(1)}, s c_{j}^{(2)}, s c_{j}^{(3)}, s c_{j}^{(4)}\right)$ & $\sim \mathrm{U}(-5,55) ; s c_{j}^{(i+1)}>s c_{j}^{(i)}$ \\
$\widetilde{m c_{j}}=\left(m c_{j}^{(1)}, m c_{j}^{(2)}, m c_{j}^{(3)}, m c_{j}^{(4)}\right)$ & $\sim \mathrm{U}(50,170) ; m c_{j}^{(i+1)}>m c_{j}^{(i)}$ \\
$\widetilde{p c}=\left(p c^{(1)}, p c^{(2)}, p c^{(3)}, p c^{(4)}\right)$ & $\sim \mathrm{U}(15,25) ; p c^{(i+1)}>p c^{i}$ \\
$\widetilde{d e}=\left(d e^{(1)}, d e^{(2)}, d e^{(3)}, d e^{(4)}\right)$ & $\sim \mathrm{U}(40,60) ; d e^{(i+1)}>d e^{i}$ \\
\hline
\end{tabular}


Table 2. PM (M) and inspection (I) activities performed in the SMMS with different objectives.

\begin{tabular}{|c|c|c|c|c|c|c|c|}
\hline \multirow{2}{*}{$\frac{\text { Objective } 1}{\text { Period }}$} & \multicolumn{3}{|c|}{ Stage } & \multirow{2}{*}{$\frac{\text { Objective2 }}{\text { Period }}$} & \multicolumn{3}{|c|}{ Stage } \\
\hline & 1 & 2 & 3 & & 1 & 2 & 3 \\
\hline 1 & $\mathrm{I}, \mathrm{M}$ & M & $\mathrm{M}$ & 1 & $\mathrm{I}, \mathrm{M}$ & $\mathrm{M}$ & M \\
\hline 2 & $\mathrm{I}, \mathrm{M}$ & M & $\mathrm{M}$ & 2 & I & & \\
\hline 3 & $\mathrm{I}, \mathrm{M}$ & M & $\mathrm{M}$ & 3 & I & I & M \\
\hline 4 & $\mathrm{I}, \mathrm{M}$ & M & $\mathrm{M}$ & 4 & I & I & \\
\hline 5 & $\mathrm{I}, \mathrm{M}$ & M & $\mathrm{M}$ & 5 & I & I & M \\
\hline 6 & $\mathrm{I}, \mathrm{M}$ & M & $\mathrm{M}$ & 6 & I & I & \\
\hline 7 & $\mathrm{I}, \mathrm{M}$ & M & $\mathrm{M}$ & 7 & I & I & M \\
\hline 8 & $\mathrm{I}, \mathrm{M}$ & M & $\mathrm{M}$ & 8 & I & I & \\
\hline 9 & $\mathrm{I}, \mathrm{M}$ & M & $\mathrm{M}$ & 9 & I & M & M \\
\hline 10 & $\mathrm{I}, \mathrm{M}$ & M & $\mathrm{M}$ & 10 & I & & \\
\hline 11 & $\mathrm{I}, \mathrm{M}$ & M & $\mathrm{M}$ & 11 & I & I & \\
\hline 12 & $\mathrm{I}, \mathrm{M}$ & M & $\mathrm{M}$ & 12 & I & I & M \\
\hline
\end{tabular}

Table 3. PM (M) and inspection (I) activities performed in the SMMS with deterministic approach.

\begin{tabular}{|c|c|c|c|}
\hline \multirow{2}{*}{$\frac{\text { Model } 2}{\text { Period }}$} & \multicolumn{3}{|c|}{ Stage } \\
\hline & 1 & 2 & 3 \\
\hline 1 & M,I & $\mathrm{M}$ & $\mathrm{M}$ \\
\hline 2 & I & I & $\mathrm{M}$ \\
\hline 3 & I & I & M \\
\hline 4 & I & I & $\mathrm{M}$ \\
\hline 5 & I & I & $\mathrm{M}$ \\
\hline 6 & I & I & M,I \\
\hline 7 & I & $\mathrm{M}$ & $\mathrm{M}$ \\
\hline 8 & I & I & M \\
\hline 9 & I & I & M \\
\hline 10 & I & & M,I \\
\hline 11 & $\mathrm{I}, \mathrm{M}$ & M & M \\
\hline 12 & $\mathrm{I}, \mathrm{M}$ & $\mathrm{M}$ & M \\
\hline
\end{tabular}

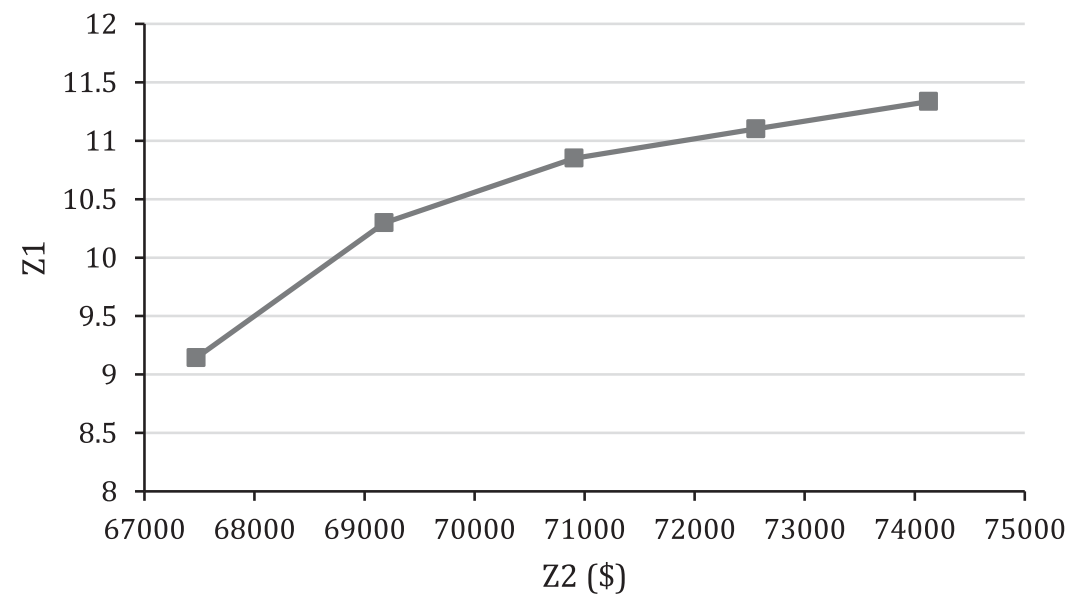

Figure 5. Obtained Pareto curve.

objective function) leads to an increase in the cost objective and vice versa. It is notable that the cost objective function has a tendency towards production of less final conforming items via minimising the use of PM activities (i.e. achieving the costefficiency). On the other hand, the first objective function has a tendency towards production of more conforming items to 
maximise the system productivity. For instance, considering the results of Model 4, when $z_{1}=11.335$ and $z_{2}=74,126.635$ (i.e. the last Pareto-optimal solution), the number of produced conforming items is 1164 and the system delivers no nonconforming item, whereas when $z_{1}=9.143$ and $z_{2}=67,471.280$ (i.e. the first Pareto-optimal solution), the number of the produced conforming items is 1045 and the system delivers 10 non-conforming items.

To show the usefulness of the applied RPP-II approach, 10 random realisations of the numerical example are first generated. For instance, if $\tilde{\xi}=\left(\xi^{(1)}, \xi^{(2)}, \xi^{(3)}, \xi^{(4)}\right)$ is a vague parameter with a trapezoidal distribution. To perform a realisation, a random number is uniformly generated between the two extreme points of the corresponding trapezoidal possibility distribution. Then, the obtained optimal solutions by the deterministic Model $2\left(x^{*}, y^{*}\right)$ and uncertain Model $3\left(x^{* *}, y^{* *}\right)$ under deterministic and nominal data, will be replaced respectively in the following linear programming models (considering realisations) that their compact forms are as follows.

$$
\begin{aligned}
& \operatorname{Min} f_{\text {real }} *^{*}+c_{\text {real }} x^{*} \\
& \text { s.t. } \\
& A x^{*} \geq d e_{\text {real }} \\
& B x^{*}=0 \\
& \operatorname{Min} f_{\text {real }} y^{* *}+c_{\text {real }} x^{* *}+\delta R^{d} \\
& \text { s.t. } \\
& A x^{* *}+R^{d} \geq d e_{\text {real }} \\
& B x^{* *}=0 \\
& R^{d} \geq 0
\end{aligned}
$$

In the linear programming model (72), $R^{d}$ is the only decision variable that specifies the violation of chance constraints under random realisation. To assess the uncertain Model 3, the mean and standard deviation of the objective function values under random realisations are employed. The results of these experiments are shown in Table 4. As can be seen, the obtained solution by the uncertain Model 3 provides lower total costs for different realisations. On average, it results in 1.1\% reduction in the total costs. In addition, applying the uncertain Model 3 achieves $0.3 \%$ reduction in the standard deviation that means the lower risk of additional imposed costs to the system.

The first objective function contains the piecewise linear segments to model the non-linear behaviour of the implied system productivity (see Section 3.1). By increasing the number of linear segments, we can achieve a more accurate estimation for the non-linear behaviour. Figure 6 depicts the obtained Pareto curve while applying different numbers of linear segments. The three-segment approximation has the lowest accuracy and employing equal and more than four segments almost causes the same result/accuracy (as it is shown for the four- and five-segment cases). Table 5 provides the obtained Pareto-optimal solutions for the different numbers of segments and shows that the average values of the objective functions for the four- and five-segment approaches are almost the same and accordingly they provide the similar accuracy for the system.

Table 4. Performance of the developed models under realisations.

\begin{tabular}{lcc}
\hline Realisation number & Model 2 & Model 3 \\
\hline 1 & $66,407.115$ & $65,705.241$ \\
2 & $74,281.224$ & $73,569.489$ \\
3 & $56,613.398$ & $55,881.472$ \\
4 & $49,663.407$ & $48,963.740$ \\
5 & $53,234.151$ & $52,687.414$ \\
6 & $66,000.509$ & $65,553.609$ \\
7 & $43,519.911$ & $43,095.735$ \\
8 & $67,653.938$ & $67,039.298$ \\
9 & $58,425.099$ & $57,735.315$ \\
10 & $53,832.192$ & $53,067.514$ \\
Average & $58,963.09$ & $58,329.88$ \\
Standard deviation & 8972.447 & 8946.283 \\
\hline
\end{tabular}




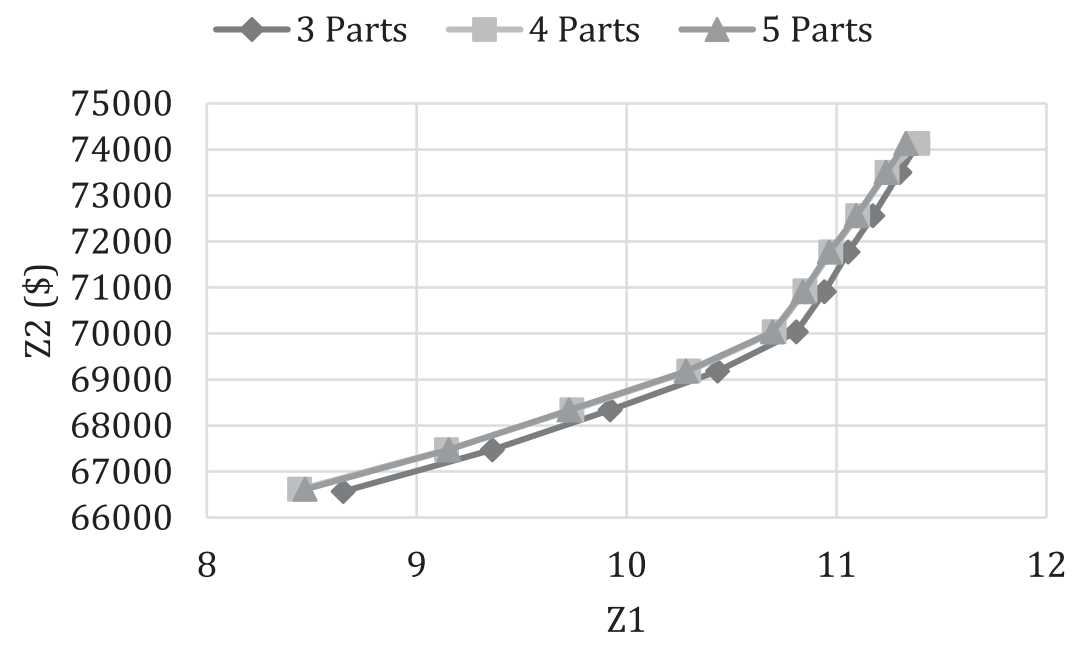

Figure 6. Pareto curve while applying different numbers of segments.

Table 5. Obtained Pareto solutions while considering different numbers of segments.

\begin{tabular}{|c|c|c|c|c|c|c|}
\hline & \multicolumn{2}{|c|}{ Three-segment } & \multicolumn{2}{|c|}{ Four-segment } & \multicolumn{2}{|c|}{ Five-segment } \\
\hline & $\mathrm{Z1}$ & $\mathrm{Z} 2$ & $\mathrm{Z1}$ & $\mathrm{Z} 2$ & $\mathrm{Z1}$ & $\mathrm{Z} 2$ \\
\hline \multirow[t]{10}{*}{ Pareto solutions } & 11.388 & $74,126.64$ & 11.388 & $74,126.64$ & 11.33 & $74,126.64$ \\
\hline & 11.302 & $73,498.81$ & 11.241 & $73,498.81$ & 11.236 & $73,498.81$ \\
\hline & 11.173 & $72,557.07$ & 11.101 & $72,557.07$ & 11.094 & $72,557.07$ \\
\hline & 11.055 & $71,766.05$ & 10.973 & $71,766.06$ & 10.965 & $71,766.05$ \\
\hline & 10.942 & $70,905.91$ & 10.85 & $70,925.18$ & 10.841 & $70,905.91$ \\
\hline & 10.808 & $70,033.3$ & 10.704 & $70,033.3$ & 10.695 & $70,033.3$ \\
\hline & 10.433 & $69,179.63$ & 10.297 & $69,179.63$ & 10.284 & $69,179.63$ \\
\hline & 9.921 & $68,336.2$ & 9.74 & $68,336.2$ & 9.726 & $68,336.2$ \\
\hline & 9.36 & $67,471.28$ & 9.143 & $67,471.28$ & 9.153 & $67,471.28$ \\
\hline & 8.65 & $66,566.81$ & 8.443 & $66,610.86$ & 8.468 & $66,611.74$ \\
\hline Mean & 10.875 & $70,469.6$ & 10.777 & $70,479.24$ & 10.768 & $70,469.6$ \\
\hline
\end{tabular}

\subsection{Model validation: a real case study}

A real case study within the car manufacturing industry in France is investigated in this section to validate the presented model and to shows the effectiveness and significance of the contributions of this paper which are the introduction of the implied system productivity objective function and the application of the RPP-II approach for dealing with the uncertainty of the cost components and demand amount.

Figure 7(a) illustrates the solid frame of the considered product and its 15 QCs are indicated in Figure 7(b). This product is manufactured by a plant in the supply chain of 'Renault Groupe'. The 15 QCs are processed along 15 different manufacturing stages. The data of the case study are gathered regarding the proposed deterministic Model 2 and uncertain Model 3 and are available upon request. The possibility distribution of vague parameters is estimated through a focus group of firm's managers and field experts. The four prominent values of each trapezoidal fuzzy number are specified regarding the available data and their knowledge. Similarly, the value of $\gamma$ and $\delta$ are considered 0.2 and 100, respectively. In addition, the planning horizon for this SMMS is 12 periods (i.e. 12 months) and the demand amount is estimated $\widetilde{d e}=(550,600,630,680)$.

To show the advantage of the obtained solution by the RPP-II technique, a similar experiment to that which was done in Section 4.1 is performed here (see Table 6). According to Table 6, the obtained solution for the deterministic Model 2 is feasible just for $50 \%$ of the realisations. Hence, it can be concluded that applying Model 2 imposes a huge feasibility risk to the system.

Tables 7 and 8 depict the obtained optimal solution by the deterministic Model 2 and uncertain Model 3, respectively. As can be seen, the uncertain Model 3's optimum solution costs 13.46\% more than the deterministic Model 2's; however as mentioned before, it can respond to all the realisations and decreases the feasibility risk around $50 \%$. 


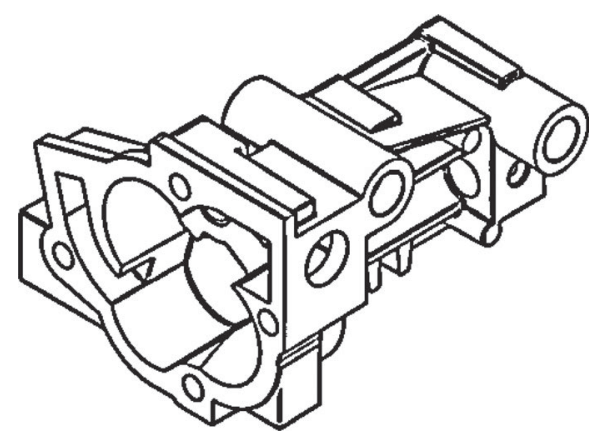

(a) Solid frame

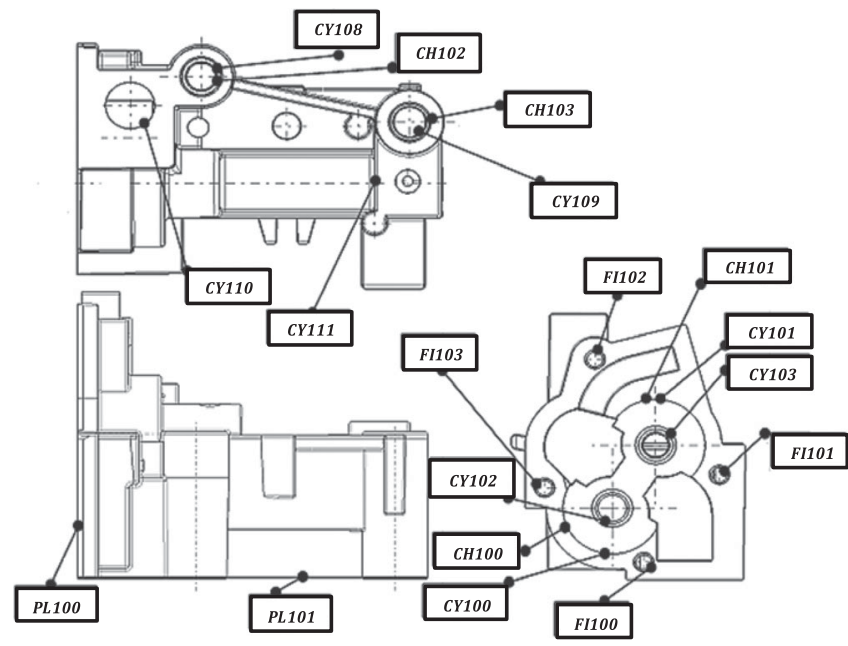

(b) QCs of the oil pump housing

Figure 7. Solid frame and QCs of the oil pump housing. (a) Solid frame. (b) QCs of the oil pump housing.

Table 6. Performance of the developed models under realisations.

\begin{tabular}{lcc}
\hline Realisation number & Model 2 & Model 3 \\
\hline 1 & $54,061.998$ & $54,859.966$ \\
2 & Infeasible & $56,798.442$ \\
3 & Infeasible & $52,771.405$ \\
4 & $54,964.901$ & $55,742.102$ \\
5 & $53,326.721$ & $54,014.608$ \\
6 & Infeasible & $53,376.714$ \\
7 & $54,229.969$ & $54,841.489$ \\
8 & Infeasible & $56,148.684$ \\
9 & $53,199.339$ & $53,796.880$ \\
10 & Infeasible & $53,556.824$ \\
\hline
\end{tabular}

The RPP-II approach contains two technical parameters, whose importance in the considered case study needs to be analysed. To this aim, a sensitivity analysis study on these two parameters is performed.

The importance/weight of the minimisation of the difference between the expected total cost and the worst-case cost against the other terms in the objective (53) is represented by $\gamma$. Table 9 shows the value of this difference and the obtained confidence level of the chance constraint (i.e. $\alpha$ ) regarding the different applied values for $\gamma$. As can be seen, the uncertain Model 3 has the best performance when the value of $\gamma$ is considered equal to 0.5. Because the difference between the expected total cost and the worst-case cost is minimum, (which means the least risk of imposed additional cost in the worst case) while the obtained confidence level of chance constraint is maximum (no risk). On the other side, the expected total cost and the worst-case cost have the lowest amount that confirms the cost-efficiency of this value $(\gamma=0.5)$. 
Table 7. Places and periods in which inspection (I) and PM (M) activities are done by using the deterministic Model 2.

\begin{tabular}{|c|c|c|c|c|c|c|c|c|c|c|c|c|c|c|c|c|}
\hline & & \multicolumn{15}{|c|}{ Stage } \\
\hline & & 1 & 2 & 3 & 4 & 5 & 6 & 7 & 8 & 9 & 10 & 11 & 12 & 13 & 14 & 15 \\
\hline \multirow[t]{12}{*}{ Period } & 1 & \multirow[t]{12}{*}{ M } & \multirow[t]{12}{*}{$\mathrm{M}$} & \multirow[t]{12}{*}{ M } & M,I & M & \multirow[t]{2}{*}{ M } & \multirow[t]{8}{*}{ M } & \multirow[t]{12}{*}{ M } & M & $\mathrm{M}$ & $\mathrm{M}$ & $\mathrm{M}$ & M & M & $\mathrm{M}$ \\
\hline & 2 & & & & I & M & & & & I & $\mathrm{M}$ & $\mathrm{M}$ & $\mathrm{M}$ & M & M & $\mathrm{M}$ \\
\hline & 3 & & & & I & & M & & & I & $\mathrm{M}$ & $\mathrm{M}$ & $\mathrm{M}$ & M & M & $\mathrm{M}$ \\
\hline & 4 & & & & I & & I & & & I & $\mathrm{M}$ & M & M & M & M & M \\
\hline & 5 & & & & I & & I & & & I & $\mathrm{M}$ & I & $\mathrm{M}$ & $\mathrm{M}$ & M & $\mathrm{M}$ \\
\hline & 6 & & & & I & & I & & & I & & & M & I & M & $\mathrm{M}$ \\
\hline & 7 & & & & I & & I & & & M,I & $\mathrm{M}$ & I & & & M,I & M \\
\hline & 8 & & & & I & & I & & & I & & & I & M,I & & M,I \\
\hline & 9 & & & & I & & I & I & & I & & I & & $\mathrm{M}$ & I & $\mathrm{M}$ \\
\hline & 10 & & & & I & & M,I & I & & I & $\mathrm{M}$ & I & & & & M,I \\
\hline & 11 & & & & I & & I & I & & M,I & & I & $\mathrm{M}$ & M & M,I & $\mathrm{M}$ \\
\hline & 12 & & & & I & & I & I & & M,I & I & $\mathrm{M}$ & $\mathrm{M}$ & M & M & M \\
\hline
\end{tabular}

Table 8. Places and periods in which inspection (I) and PM (M) activities are done by using the uncertain Model 3.

\begin{tabular}{|c|c|c|c|c|c|c|c|c|c|c|c|c|c|c|c|c|}
\hline & \multicolumn{15}{|c|}{ Stage } \\
\hline & & 1 & 2 & 3 & 4 & 5 & 6 & 7 & 8 & 9 & 10 & 11 & 12 & 13 & 14 & 15 \\
\hline \multirow[t]{12}{*}{ Period } & 1 & \multirow[t]{10}{*}{ M } & \multirow[t]{12}{*}{ M } & \multirow[t]{12}{*}{ M } & M,I & \multirow[t]{3}{*}{ M } & \multirow[t]{2}{*}{ M } & \multirow[t]{3}{*}{ M } & M & \multirow[t]{4}{*}{ M } & M & M & M & M & M & $\mathrm{M}$ \\
\hline & 2 & & & & I & & & & I & & $\mathrm{M}$ & $\mathrm{M}$ & $\mathrm{M}$ & $\mathrm{M}$ & $\mathrm{M}$ & $\mathrm{M}$ \\
\hline & 3 & & & & I & & I & & I & & M & M & M & M & M & $\mathrm{M}$ \\
\hline & 4 & & & & I & \multirow[t]{9}{*}{ M } & & $\mathrm{M}$ & I & & $\mathrm{M}$ & $\mathrm{M}$ & $\mathrm{M}$ & $\mathrm{M}$ & $\mathrm{M}$ & $\mathrm{M}$ \\
\hline & 5 & & & & I & & I & & I & $\mathrm{M}$ & & & & I & $\mathrm{M}$ & $\mathrm{M}$ \\
\hline & 6 & & & & I & & I & & I & $\mathrm{M}$ & & & I & & $\mathrm{M}$ & M,I \\
\hline & 7 & & & & I & & I & & I & & & & I & $\mathrm{M}$ & & M,I \\
\hline & 8 & & & & I & & I & & M,I & & $\mathrm{M}$ & $\mathrm{M}$ & $\mathrm{M}$ & & I & $\mathrm{M}$ \\
\hline & 9 & & & & I & & I & & & & M,I & & $\mathrm{M}$ & & $\mathrm{M}$ & M,I \\
\hline & 10 & & & & I & & I & M & $\mathrm{M}$ & M & & M,I & M & & M & M,I \\
\hline & 11 & $\mathrm{I}$ & & & I & & I & M & & $\mathrm{M}$ & $\mathrm{M}$ & M & M & I & $\mathrm{M}$ & $\mathrm{M}$ \\
\hline & 12 & & & & I & & M & I & & M & & M & M & M,I & M & $\mathrm{M}$ \\
\hline
\end{tabular}

Table 9. Impact of $\gamma$ on the expected total cost, worstcase cost, and confidence level.

\begin{tabular}{lcccl}
\hline$\gamma$ & $z_{1}^{\max }$ & $E\left[z_{1}\right]$ & $z_{1}^{\max }-E\left[z_{1}\right]$ & $\alpha$ \\
\hline 0.01 & $69,732.01$ & $57,181.39$ & $12,550.62$ & 1 \\
0.1 & $69,908.67$ & $57,340.77$ & $12,567.9$ & 1 \\
0.2 & $69,532.05$ & $57,030.65$ & $12,501.4$ & 1 \\
$\mathbf{0 . 5}$ & $\mathbf{6 9 , 4 1 1 . 4 1}$ & $\mathbf{5 6 , 9 3 0 . 7 6}$ & $\mathbf{1 2 , 4 8 0 . 6 5}$ & $\mathbf{1}$ \\
1 & $69,986.34$ & $57,399.41$ & $12,586.93$ & 0.996 \\
10 & $69,789.65$ & $57,250.43$ & $12,539.22$ & 0.976 \\
100 & $69,784.04$ & $57,265.72$ & $12,518.32$ & 0.5 \\
\hline
\end{tabular}

Table 10. Performance of the uncertain Model 3 while considering different values for $\delta$.

\begin{tabular}{lcccc}
\hline$\delta$ & \multicolumn{1}{c}{$z_{1}^{\max }$} & \multicolumn{1}{c}{$E\left[z_{1}\right]$} & $z_{1}^{\max }-E\left[z_{1}\right]$ & $\alpha$ \\
\hline 1 & $69,684.95$ & $57,160.46$ & $12,524.49$ & 0.526 \\
10 & $69,885.69$ & $57,329.88$ & $12,555.8$ & 0.954 \\
50 & $69,603.77$ & $57,069.6$ & $12,534.18$ & 0.889 \\
$\mathbf{1 0 0}$ & $\mathbf{6 9 , 4 1 1 . 4 1}$ & $\mathbf{5 6 , 9 3 0 . 7 6}$ & $\mathbf{1 2 , 4 8 0 . 6 5}$ & $\mathbf{1}$ \\
200 & $69,773.81$ & $57,234.89$ & $12,538.91$ & 1 \\
300 & $69,876.18$ & $57,325.14$ & $12,551.04$ & 1 \\
500 & $69,974.67$ & $57,397.28$ & $12,577.38$ & 1 \\
\hline
\end{tabular}




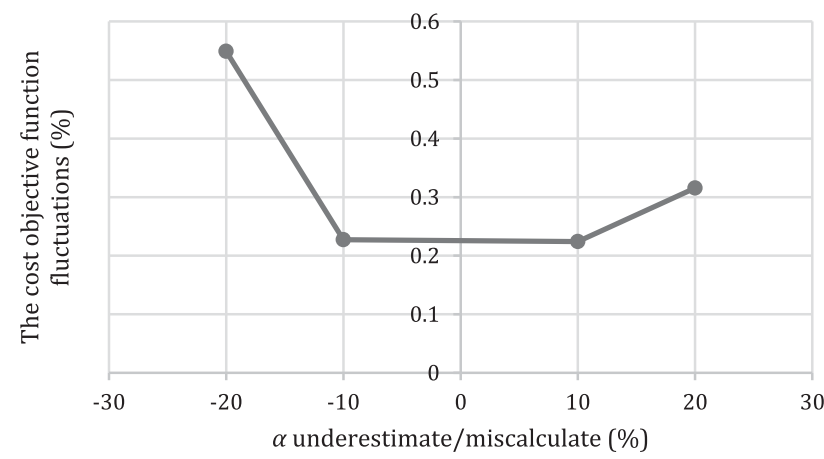

(a) Sensitivity of the total cost objective function on $\alpha$

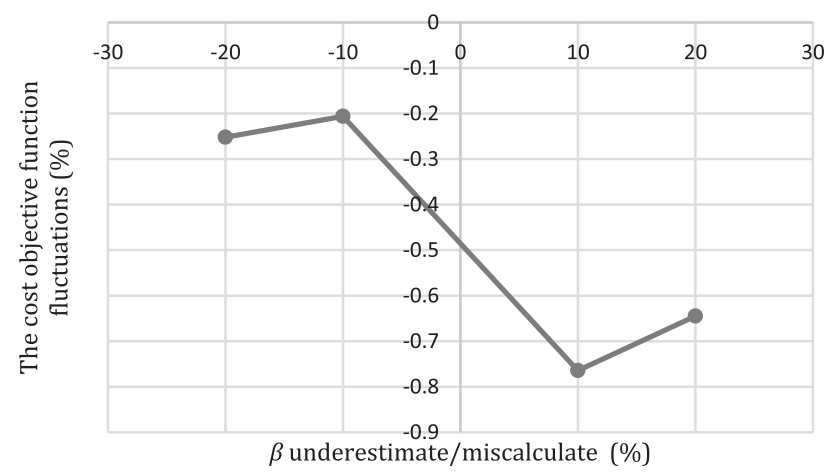

(b) Sensitivity of the total cost objective function on $\beta$

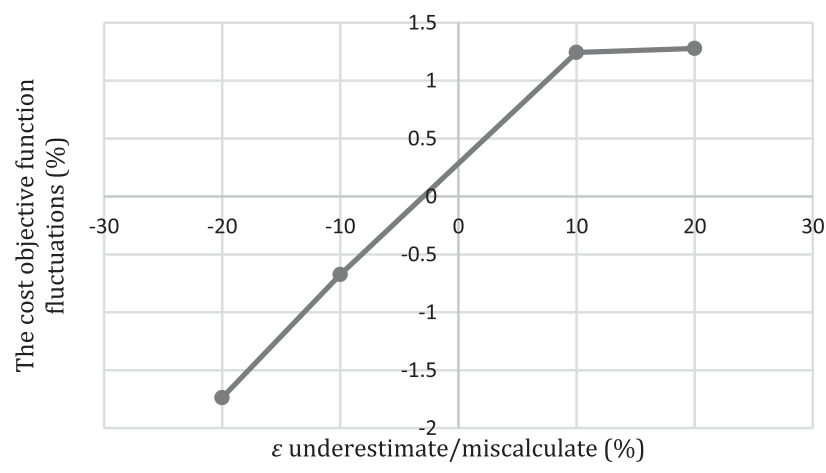

(c) Sensitivity of the total cost objective function on $\varepsilon$

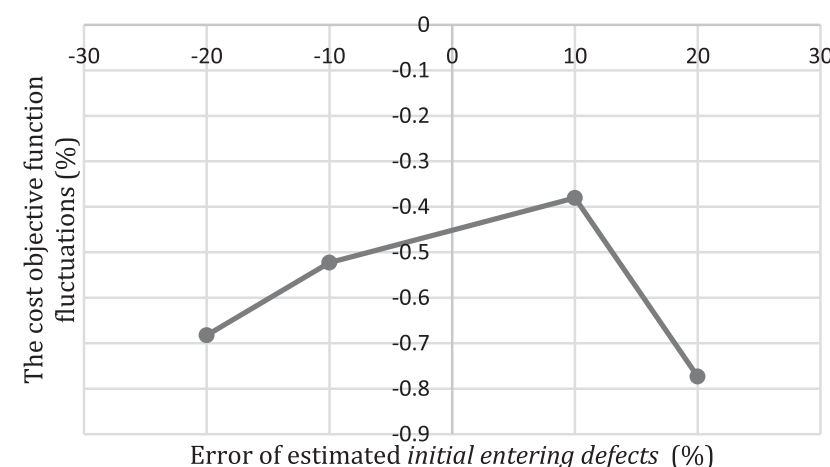

(d) Sensitivity of the total cost objective function on $\varepsilon_{0}$

Figure 8. Sensitivity of the total cost objective function on the known parameter. 
The third term of the objective function (53) (i.e. $\left.\delta\left[d e^{(4)}-(1-\theta) d e^{(3)}-\theta d e^{(4)}\right]\right)$ specifies the confidence level of the demand chance constraint, in which $\delta$ is the penalty unit of possible violation of the constraint containing vague parameter and $\left[d e^{(4)}-(1-\theta) d e^{(3)}-\theta d e^{(4)}\right]$ shows the difference between the worst-case value of imprecise parameter and the value that is applied in the demand chance constraint. Hence, the feasibility robustness of the solution vector is controlled by this term. It is notable that $\delta$ is not just a meaningless parameter and theoretical, rather the value of penalty can be properly specified according to the application context (Pishvaee, Razmi, and Torabi 2012). For example, in our case study, the value of $\delta$ can be considered as the penalty of shortage that is known as a popular parameter in the context of manufacturing.

Table 10 shows the performance of the uncertain Model 3 regarding different values of $\delta$. The best value considered for $\delta$ is 100 because the uncertain Model 3 reaches not only the highest confidence level $(\alpha=1)$, but also the least values for average and worst-case total cost. In other words, the solution obtained by considering $\delta=100$, is the most risk-averse and cost-efficient solution.

By employing the RPP-II, this paper tries to handle the uncertainty about cost components and demand amount that are rooted in the external reasons to provide a robust solution. However, the rest of the parameters may undergo fluctuations because of the internal reasons. Figure 8 shows the impacts of these fluctuations on the total cost objective function. As can be seen, even if the system undergoes $20 \%$ fluctuations for the $\alpha, \beta, \varepsilon$, and $\varepsilon_{0}$ parameter, the changes of the objective function are not more than $1.7 \%$, which is negligible. Hence, it can be concluded that the obtained solution by the uncertain Model 3 is remained robust under $20 \%$ miscalculation/underestimate of the deterministic parameters.

In this section, we utilise the four-segment linear approximation and do not go further (e.g. six-segment and more) because of the obtained same accuracy after applying the four-segment approximation. It is notable that each run for obtaining a Pareto-optimal solution takes time around 24-36 hours and the DM is satisfied to select between these fives; however, one may prefer more options to select and obtain more Pareto solutions as it is possible by consuming more time.

Figure 9 and Table 11 show the obtained Pareto curve for the case study. As can be seen, by improving the system productivity, the total cost is getting worse. Since there is no optimal solution for the multi-objective problems, one of the Pareto-optimal solutions should be selected based on the DM's preference. The DM prefers the third Pareto solution (see Table 12 for the details of the solution) because the company can increase its system productivity by around $15 \%$ through investing about $4 \%$ more. The fourth solution also can be a preferable solution for the DM, but the company is not able to invest $5 \%$ and more.

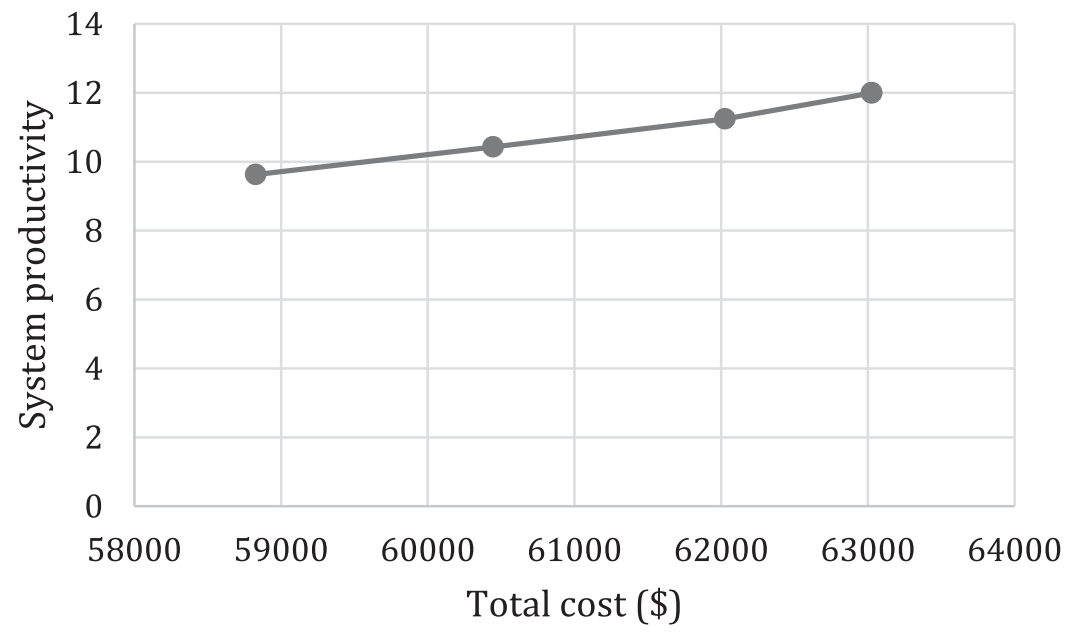

Figure 9. Obtained Pareto curve for the case study.

Table 11. Pareto-optimal solutions for the case study.

\begin{tabular}{lccc}
\hline No & Epsilon & Total cost $(\$)$ & System productivity \\
\hline 1 & $72,330.661$ & $63,027.01$ & 11.995 \\
2 & $70,554.491$ & $63,027.01$ & 11.995 \\
3 & $68,778.322$ & $62,026.94$ & 11.244 \\
4 & $67,002.152$ & $60,446.32$ & 10.429 \\
5 & $65,225.983$ & $58,829.67$ & 9.6310 \\
\hline
\end{tabular}


Table 12. Third Pareto-optimal solution: places and periods, in which inspection (I) activities and PM (M) activities are done.

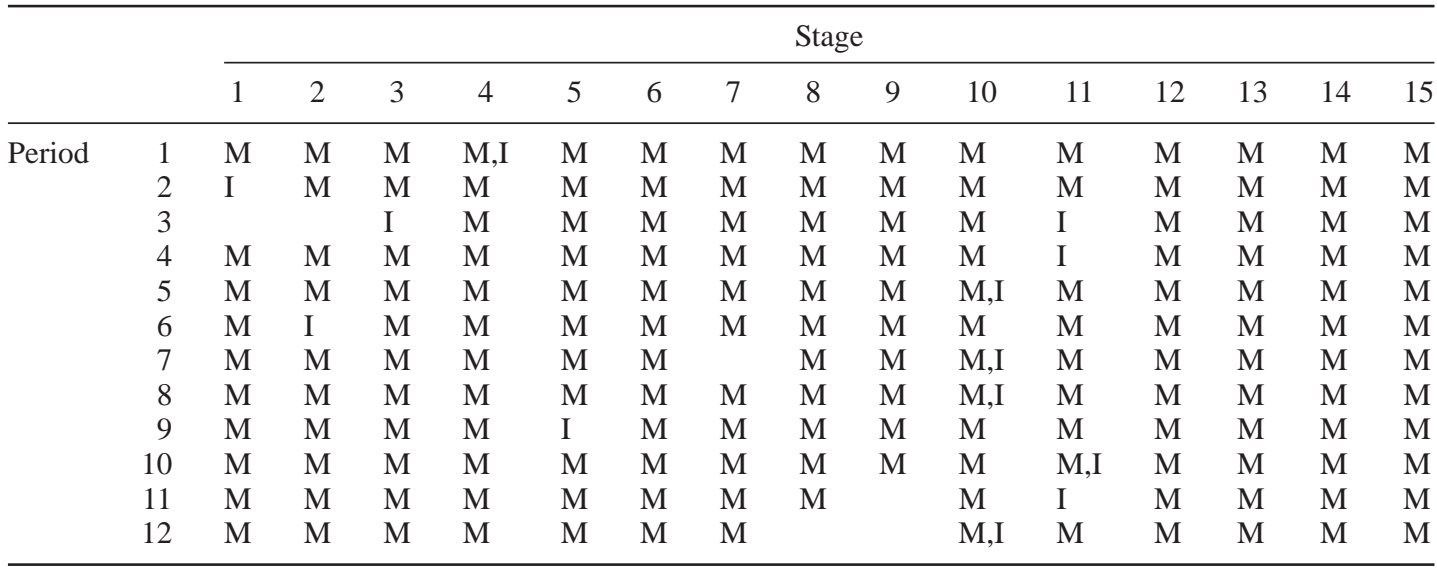

\section{Conclusion and future research direction}

This work has presented a robust possibilistic, bi-objective, MILP model for the integrated planning problem of the PM and part quality inspection activities in deteriorating SMMSs. The proposed model specifies the optimum time and place for the PM and part quality inspection activities while simultaneously optimising two objectives: (1) system productivity (2) total cost. A non-linear utility function of produced conforming items was defined as a measure of system productivity and a piecewise linear approximation approach has been employed to deal with the non-linearity. This new objective function makes an opportunity for the manufacturing companies to establish a trade-off between system productivity and total cost which would be a great advantage in today's competitive environment. In addition, a RPP approach has been used to handle the uncertainty which is rooted in the dynamic nature of the problem. Finally, a numerical example and a real case study have been investigated for the validation and verification purposes. The results show that the robust possibilistic approach decreases the variance of the total cost in any realisation of uncertain parameters in comparison to the deterministic approach. Hence, through applying the robust possibilistic approach, the fluctuations in the cost components cannot impose a huge unpredictable cost to the company.

In this paper, it was assumed that the cost parameters and demand amount are uncertain because of the market fluctuation. However, the other parameters of the problem (e.g. DPR, error probability of inspection activities and a repairable fraction of the rejected items) are uncertain because of the dynamic nature of different production stages. Therefore, considering these uncertainties and immunising the obtained solution against them is an interesting direction for future studies.

Most of the actual manufacturing systems follow the non-serial and convergent structures. In these structures, mathematically tracking the root defects after each stage is too complex. So, investigating the challenging IPQIPMP problem in the context of a non-serial or convergent structure is a big step to help a bigger number of manufacturing systems.

In recent years, some scientists have attempted to design production systems that are psychologically consistent with operators (e.g. see Azadeh et al. 2015a, 2017). This consistency impacts the accuracy of the operators (Rezaei-Malek et al. 2017). Since operators do an inspection process in the most of production systems, it would be interesting to integrate this new concept into the IPQIPMP problem.

Reduction of inventory has a positive consequence on the product quality because quality defects are determined earlier and are not propagated throughout the manufacturing system (Colledani et al. 2014). In this regard, some researchers have attempted to consider production and PM planning in conjunction with the determination of inventory-related decisions (e.g. see Liu, Wang, and Peng 2015). However, incorporation of the inventory-related issues into the IPQIPMP problem is one of the research gaps that would be interesting as a future research direction.

\section{Acknowledgements}

The first author would like to acknowledge the Campus France and France Embassy in Tehran for their financial support under the scholarship number 906221A.

\section{Disclosure statement}

No potential conflict of interest was reported by the authors. 


\section{ORCID}

Mohammad Rezaei-Malek (D) http://orcid.org/0000-0002-0772-2255

Ali Siadat (D) http://orcid.org/0000-0002-8406-1892

Jean-Yves Dantan (D) http://orcid.org/0000-0002-0491-8391

Reza Tavakkoli-Moghaddam (D) http://orcid.org/0000-0002-6757-926X

\section{References}

Azadeh, A., M. Ravanbakhsh, M. Rezaei-Malek, M. Sheikhalishahi, and A. Taheri-Moghaddam. 2017. "Unique NSGA-II and MOPSO Algorithms for Improved Dynamic Cellular Manufacturing Systems Considering Human Factors." Applied Mathematical Modelling 48: 655-672.

Azadeh, A., M. Rezaei-Malek, F. Evazabadian, and M. Sheikhalishahi. 2015a. "Improved Design of CMS by Considering Operators Decision-making Styles." International Journal of Production Research 53 (11): 3276-3287.

Azadeh, A., M. S. Sangari, E. Sangari, and S. Fatehi. 2015b. "A Particle Swarm Algorithm for Optimising Inspection Policies in Serial Multistage Production Processes with Uncertain Inspection Costs.” International Journal of Computer Integrated Manufacturing 28 (7): 766-780.

Ben-Daya, M., and M. Rahim. 2001. "Integrated Production, Quality and Maintenance Models, an Overview." In Integrated Models in Production Planning, Inventory, Quality and Maintenance, edited by M. Rahim and M. Ben-Daya, 3-28. New York: Kluwer Academic.

Colledani, M., T. Tolio, A. Fischer, B. Iung, G. Lanza, R. Schmitt, and J. Váncza. 2014. "Design and Management of Manufacturing Systems for Production Quality." CIRP Annals - Manufacturing Technology 63 (2): 773-796.

Eppen, G. D., and G. E. Hurst. 1974. "Optimal Location of Inspection Stations in a Multistage Production Process." Management Science 20 (8): 1194-1200.

Galante, G., and G. Passannanti. 2007. "Integrated Approach to Part Scheduling and Inspection Policies for a Job Shop Manufacturing System.” International Journal of Production Research 45 (22): 5177-5198.

Gunter, S. I., and L. A. Swanson. 1985. "Inspector Location in Convergent Production Lines." International Journal of Production Research 23 (6): 1153-1169.

Kakade, V., J. F. Valenzuela, and J. S. Smith. 2004. “An Optimization Model for Selective Inspection in Serial Manufacturing Systems.” International Journal of Production Research 42 (18): 3891-3909.

Kim, J., and S. B. Gershwin. 2008. "Analysis of Long Flow Lines with Quality and Operational Failures." IIE Transactions 40 (3): 284-296.

Kogan, K., and T. Raz. 2002. “Optimal Allocation of Inspection Effort Over a Finite Planning Horizon.” IIE Transactions 34 (6): $515-527$.

Lindsay, G. N., and A. B. Bishop. 1964. “Allocation of Screening Inspection Effort-A Dynamic-Programming Approach.” Management Science 10 (2): 342-352.

Liu, X., W. Wang, and R. Peng. 2015. "An Integrated Production, Inventory and Preventive Maintenance Model for a Multi-Product Production System.” Reliability Engineering and System Safety 137: 76-86.

Mandroli, S. S., A. K. Shrivastava, and Y. Ding. 2006. "A Survey of Inspection Strategy and Sensor Distribution Studies in Discrete-Part Manufacturing Processes.” IIE Transactions 38 (4): 309-328.

Mavrotas, G. 2009. "Effective Implementation of the $\varepsilon$-Constraint Method in Multi-Objective Mathematical Programming Problems." Applied Mathematics and Computation 213 (2): 455-465.

Mohammadi, M., J. Y. Dantan, A. Siadat, and R. Tavakkoli-Moghaddam. 2018. “A Bi-Objective Robust Inspection Planning Model in a Multi-Stage Serial Production System.” International Journal of Production Research 56 (4): 1432-1457.

Mohammadi, M., A. Siadat, J.-Y. Dantan, and R. Tavakkoli-Moghaddam. 2015. "Mathematical Modelling of a Robust Inspection Process Plan: Taguchi and Monte Carlo Methods." International Journal of Production Research 53 (7): 2202-2224.

Narahari, Y., and L. M. Khan. 1996. "Modeling Reentrant Manufacturing Systems with Inspection Stations." Journal of Manufacturing Systems 15 (6): 367-378.

Park, H. Y., H. E. Park, and A. C. Ntuen. 1988. "A Study for Optional Inspection Policies in a Flexible Manufacturing Cell.” Computer and Industrial Engineering 15 (1-4): 307-314.

Penn, M., and T. Raviv. 2007. “Optimizing the Quality Control Station Configuration.” Naval Research Logistics 54 (3): $301-314$.

Penn, M., and T. Raviv. 2008. “A Polynomial Time Algorithm for Solving a Quality Control Station Configuration Problem.” Discrete Applied Mathematics 156 (4): 412-419.

Pishvaee, M. S., J. Razmi, and S. Torabi. 2012. "Robust Possibilistic Programming for Socially Responsible Supply Chain Network Design: A New Approach.” Fuzzy Sets and Systems 206: 1-20.

Pruzan, P. M., and J. T. R. Jackson. 1967. “A Dynamic Programming Application in Production Line Inspection.” Techno-metrics 9 (1): 73-81.

Raghavachari, M., and G. Tayi. 1991. "Inspection Configuration and Reprocessing Decisions in Serial Production Systems.” International Journal of Production Research 29 (5): 897-911. 
Raz, T. 1986. “A Survey of Models for Allocating Inspection Effort in Multistage Production Systems.” Journal of Quality Technology 18 (4): 239-247.

Raz, T., Y. T. Herer, and A. Grosfeld-Nir. 2000. “Economic Optimization of Off-Line Inspection.” IIE Transactions 32 (3): $205-217$.

Rezaei-Malek, M., M. Mohammadi, J.-Y. Dantan, A. Siadat, and R. Tavakkoli-Moghaddam. 2018b. "A Review on Optimization of Part Quality Inspection Planning in a Multi-Stage Manufacturing System.” International Journal of Production Research. doi:10.1080/00207543.2018.1464231.

Rezaei-Malek, M., A. Siadat, J.-Y. Dantan, and R. Tavakkoli-Moghaddam. 2018a. "An Approximation Approach for an Integrated Part Quality Inspection and Preventive Maintenance Planning in a Nonlinear Deteriorating Serial Multi-Stage Manufacturing System.” IFAC-PapersOnLine 51 (11): 270-275.

Rezaei-Malek, M., R. Tavakkoli-Moghaddam, N. Cheikhrouhou, and A. Taheri-Moghaddam. 2016. "An Approximation Approach to a Trade-Off among Efficiency, Efficacy, and Balance for Relief Pre-Positioning in Disaster Management." Transportation Research Part E: Logistics and Transportation Review 93: 485-509.

Rezaei-Malek, M., R. Tavakkoli-Moghaddam, A. Siadat, and J.-Y. Dantan. 2017. "Integrated Preventive Maintenance and Inspection Planning in a Deteriorating Serial Multi-stage Manufacturing System.” In Proceedings of the 7th IESM Conference, Vol. 2, 352357. Saarbrücken, October 11-13.

Rezaei-Malek, M., R. Tavakkoli-Moghaddam, A. Siadat, and J.-Y. Dantan. 2018c. "A Novel Model for the Integrated Planning of Part Quality Inspection and Preventive Maintenance in a Linear-Deteriorating Serial Multi-Stage Manufacturing System.” The International Journal of Advanced Manufacturing Technology 96 (9-12): 3633-3650.

Rossit, D. G., F. A. Tohmé, M. Frutos, and D. R. Broz. 2017. "An Application of the Augmented E-Constraint Method to Design a Municipal Sorted Waste Collection System.” Decision Science Letters 6 (4): 323-336.

Sadegheih, A. 2007. "Sequence Optimization and Design of Allocation Using GA and SA." Applied Mathematics and Computation 186 (2): 1723-1730.

Shetwan, A. G., V. I. Vitanov, and B. Tjahjono. 2011. "Allocation of Quality Control Stations in Multistage Manufacturing Systems." Computers and Industrial Engineering 60 (4): 473-484.

Shiau, Y. R., M. H. Lin, and W. C. Chuang. 2007. "Concurrent Process/Inspection Planning for a Customized Manufacturing System Based on Genetic Algorithm." The International Journal of Advanced Manufacturing Technology 33 (7-8): 746-755.

Tayi, G., and D. Ballou. 1988. “An Integrated Production-Inventory Model with Reprocessing and Inspection." International Journal of Production Research 26 (8): 1299-1315.

White, L. S. 1966. “The Analysis of a Simple Class of Multi-Stage Inspection Plans.” Management Science 12 (9): $685-693$.

White, L. S. 1969. "Shortest Route Models for the Allocation of Inspection Effort on a Production Line." Management Science 15 (5): 249-259.

Williams, H. P. 2013. Model Building in Mathematical Programming. West Sussex: John Wiley.

Yum, B. J., and E. D. McDowellj. 1987. "Optimal Inspection Policies in a Serial Production System Including Scrap Rework and Repair: An MILP Approach." International Journal of Production Research 25 (10): 1451-1464.

Zahiri, B., R. Tavakkoli-Moghaddam, and M. Rezaei-Malek. 2016. "An MCDA-DEA Approach for Mixed-Model Assembly Line Balancing Problem Under Uncertainty.” Journal of Intelligent \& Fuzzy Systems 30 (5): 2737-2748. 